S. Goto, K. Nishida and Y. Shimoda

Nagoya Math. J.

Vol. 124 (1991), 99-132

\title{
TOPICS ON SYMBOLIC REES ALGEBRAS FOR SPACE MONOMIAL CURVES
}

\author{
SHIRO GOTO, KOJI NISHIDA AND YASUHIRO SHIMODA ${ }^{1}$
}

\section{§1. Introduction}

Let $A$ be a regular local ring of $\operatorname{dim} A=3$ and $\mathfrak{p}$ a prime ideal in $A$ of $\operatorname{dim} A / \mathfrak{p}=1$. We put $R_{s}(\mathfrak{p})=\sum_{n \geq 0} \mathfrak{p}^{(n)} t^{n}$ (here $t$ denotes an indeterminate over $A$ ) and call it the symbolic Rees algebra of $\mathfrak{p}$. With this notation the authors $[5,6]$ investigated the condition under which the $A$-algebra $R_{s}(\mathfrak{p})$ is Cohen-Macaulay and gave a criterion for $R_{s}(\mathfrak{p})$ to be a Gorenstein ring in terms of the elements $f$ and $g$ in Huneke's condition [11, Theorem 3.1] of $R_{s}(\mathfrak{p})$ being Noetherian. They furthermore explored the prime ideals $\mathfrak{p}=\mathfrak{p}\left(n_{1}, n_{2}, n_{3}\right)$ in the formal power series ring $A=$ $k \llbracket X, Y, Z \rrbracket$ over a field $k$ defining space monomial curves $X=t^{n_{1}}, Y=t^{n_{2}}$ and $Z=t^{n_{3}}$ with $\operatorname{GCD}\left(n_{1}, n_{2}, n_{3}\right)=1$ and proved that $R_{s}(\mathfrak{p})$ are Gorenstein rings for certain prime ideals $\mathfrak{p}=\mathfrak{p}\left(n_{1}, n_{2}, n_{3}\right)$. In the present research, similarly as in $[5,6]$, we are interested in the ring-theoretic properties of $R_{s}(\mathfrak{p})$ mainly for $\mathfrak{p}=\mathfrak{p}\left(n_{1}, n_{2}, n_{3}\right)$ and the results of $[5,6]$ will play key roles in this paper.

However, the problem whether $R_{s}(\mathfrak{p})$ is a finitely generated $A$-algebra is more fundamental and as far as the authors know, it remains open even for the general space monomial curves $\mathfrak{p}=\mathfrak{p}\left(n_{1}, n_{2}, n_{3}\right)$. And the problem was one of the motivations of J. Herzog and B. Ulrich to develop a theory on self-linked curve singularities [9], where they gave a criterion for $R_{s}(\mathfrak{p})$ to be generated in degree two and proved that $\mathfrak{p}$ is self-linked and $R_{s}(\mathfrak{p})$ is a Gorenstein ring, once $R_{s}(\mathfrak{p})=A\left[\mathfrak{p} t, \mathfrak{p}^{(2)} t^{2}\right]$. In our paper we shall succeed the research of Herzog and Ulrich [9]; of course our target is the next step, that is, the study of the $A$-subalgebra $A\left[\mathfrak{p} t, \mathfrak{p}^{(2)} t^{2}\right.$, $\left.\mathfrak{p}^{(3)} t^{3}\right]$ of $R_{s}(\mathfrak{p})$. There are two purposes: one is to find the conditions under which $R_{s}(\mathfrak{p})$ is generated in degree three and the other one is to

Received October 22, 1990.

1 The authors are partially supported by Grant-in-Aid for Co-operative Research. 
see what kind of ring-theoretic properties $R_{s}(\mathfrak{p})$ possesses, when $R_{s}(\mathfrak{p})=$ $A\left[\mathfrak{p} t, \mathfrak{p}^{(2)} t^{2}, \mathfrak{p}^{(3)} t^{3}\right]$.

Now let us explain our results. Throughout the work [9] the information from [12] about $\mathfrak{p}^{(2)}$ played an important role and we will briefly summarize the results on $\mathfrak{p}^{(2)}$ in Sections 2 and 4 , because they are still helpful for our purposes, too. But we furthermore need an explicit structure theorem of the third symbolic powers $\mathfrak{p}^{(3)}$, which we will discuss in Section 5. The ring-theoretic properties of $R_{s}(p)$ heavily depend on whether $\mathfrak{p}$ is self-linked or not. In fact, the ring $R_{s}(\mathfrak{p})$ is necessarily Gorenstein, if $\mathfrak{p}$ is self-linked and if $R_{s}(\mathfrak{p})=A\left[\mathfrak{p} t, \mathfrak{p}^{(2)} t^{2}, \mathfrak{p}^{(3)} t^{3}\right]$. But $R_{s}(\mathfrak{p})$ cannot be Cohen-Macaulay and the characteristic of the ground field has to be 2, if $\mathfrak{p}$ is not self-linked although $R_{s}(\mathfrak{p})=A\left[\mathfrak{p} t, \mathfrak{p}^{(2)} t^{2}, \mathfrak{p}^{(3)} t^{3}\right]$. These assertions will be proved in Theorems (6.1) and (6.12), where the conditions under which the $A$-algebra $R_{s}(\mathfrak{p})$ is generated in degree three shall be summarized, too. As was proved in [9], $A\left[\mathfrak{p} t, \mathfrak{p}^{(2)} t^{2}\right]$ is a Gorenstein ring if $\mathfrak{p}$ is selflinked. However, this assertion is no more true when $\mathfrak{p}$ is not self-linked. In fact, $A\left[\mathfrak{p} t, \mathfrak{p}^{(2)} t^{2}\right]$ is not a Cohen-Macaulay ring for $\mathfrak{p}=\mathfrak{p}(13,14,17)$, while the rings $A\left[\mathfrak{p} t, \mathfrak{p}^{(2)} t^{2}\right]$ are Cohen-Macaulay and of $r\left(A\left[\mathfrak{p} t, \mathfrak{p}^{(2)} t^{2}\right]\right)=3$ for a certain large class of prime ideals $\mathfrak{p}=\mathfrak{p}\left(n_{1}, n_{2}, n_{3}\right)$ (cf. Section 3 and [9]). Thus our main purposes will be attained to by the end of Section 6 .

As a final topic we would like to include in Section 7 an answer to a question posed by Huckaba [10, Remark 2.6 (3)]. He asked the relationship between Huneke's condition [13, Theorem 1.4] of $R_{s}(\mathfrak{p})$ being Noetherian and his own condition for that. Our answer Theorem (7.1) will show that they are equivalent to each other.

In what follows let $A$ denote a 3-dimensional regular local ring with maximal ideal $\mathfrak{m}$ and $\mathfrak{p}$ a prime ideal in $A$ of $\operatorname{dim} A / \mathfrak{p}=1$. For a given ideal $I$ we denote by $\lambda(I)$ the analytic spread of $I$. Let $\ell_{A}(M)$ and $\mu_{A}(M)$ respectively denote, for each finitely generated $A$-module $M$, the length of $M$ and the number of elements in a minimal system of generators for $M$.

\section{§ 2. Preliminaries}

Let $X, Y, Z$ be a regular system of parameters for $A$ and assume that $\mathfrak{p}$ is generated by the maximal minors of a matrix $M$ of the form

$$
M=\left[\begin{array}{lll}
X^{\alpha} & Y^{\beta^{\prime}} & Z^{\gamma^{\prime}} \\
Y^{\beta} & Z^{\gamma} & X^{\alpha^{\prime}}
\end{array}\right],
$$


where $\alpha, \beta, \gamma, \alpha^{\prime}, \beta^{\prime}, \gamma^{\prime}$ are positive integers. Notice that if $\mathfrak{p}=\mathfrak{p}\left(n_{1}, n_{2}, n_{3}\right)$ and $\mu_{A}(\mathfrak{p})=3$, then the ideal $\mathfrak{p}$ satisfies the above assumption (cf. [7]).

The purpose of this section is to summarize certain results on the second symbolic powers $\mathfrak{p}^{(2)}$. To begin with we note the following

Lemma (2.1). (1) $\alpha \neq \alpha^{\prime}, \beta \neq \beta^{\prime}$ or $\gamma \neq \gamma^{\prime}$.

(2) $2 \alpha \neq \alpha^{\prime}, 2 \beta \neq \beta^{\prime}$ or $\gamma \neq \gamma^{\prime}$.

(3) If $\mathfrak{p}=\mathfrak{p}\left(n_{1}, n_{2}, n_{3}\right)$ for some positive integers $n_{1}, n_{2}$ and $n_{3}$, then $\alpha \neq 2 \alpha^{\prime}, 2 \beta \neq \beta^{\prime}$ or $2 \gamma \neq \gamma^{\prime}$.

Proof. (1) See [6, Lemma (2.3)].

(2) Suppose that $2 \alpha=\alpha^{\prime}, 2 \beta=\beta^{\prime}$ and $\gamma=\gamma^{\prime}$. Then since $\left(X^{3 \alpha}-Y^{\beta} Z^{\gamma}\right)$ $-\left(Y^{3 \beta}-X^{\alpha} Z^{r}\right)=\left(X^{\alpha}-Y^{\beta}\right)\left(X^{2 \alpha}+X^{\alpha} Y^{\beta}+Y^{2 \beta}+Z^{\gamma}\right)$, we have $X^{\alpha}-Y^{\beta} \in \mathfrak{p}$ or $X^{2 \alpha}+X^{\alpha} Y^{\beta}+Y^{2 \beta}+Z^{\gamma} \in \mathfrak{p}$. Therefore at least one of $Y^{\beta}$ and $Y^{2 \beta}+Z^{\gamma}$ must be in the ideal $(X)+\mathfrak{p}=\left(X, Z^{2 \gamma}, Y^{\beta} Z^{\gamma}, Y^{3 \beta}\right)$, which is impossible.

(3) Suppose that $\alpha=2 \alpha^{\prime}, 2 \beta=\beta^{\prime}$ and $2 \gamma=\gamma^{\prime}$. Then $Z^{3 \gamma}-X^{\alpha^{\prime}} Y^{2 \beta} \in \mathfrak{p}$ and $X^{3 \alpha^{\prime}}-Y^{\beta} Z^{2 \gamma} \in \mathfrak{p}$. Hence $3 \gamma n_{3}=\alpha^{\prime} n_{1}+2 \beta n_{2}$ and $3 \alpha^{\prime} n_{1}=\beta n_{2}+2 \gamma n_{3}$. These equations imply $\alpha^{\prime} n_{1}=\gamma n_{3}$ and so $X^{\alpha^{\prime}}-Z^{\gamma} \in \mathfrak{p}$. Thus we get $Z^{\gamma} \in$ $(X)+\mathfrak{p}=\left(X, Z^{3 \gamma}, Y^{\beta} Z^{2 \gamma}, Y^{3 \beta}\right)$, which is impossible.

After suitable permutations of the rows and columns of $M$, we may assume that $M$ is one of the following types.

(I) $\alpha \leq \alpha^{\prime}, \beta \leq \beta^{\prime}$ and $\gamma \leq \gamma^{\prime}$,

(II) $\alpha>\alpha^{\prime}, \beta<\beta^{\prime}$ and $\gamma<\gamma^{\prime}$.

We note that $\mathfrak{p}$ is self-linked (resp. not self-linked) if and only if $M$ has type (I) (resp. type (II)) (cf. [9, Corollary 1.10]). We put

$$
a=Z^{\gamma+\gamma^{\prime}}-X^{\alpha^{\prime}} Y^{\beta^{\prime}}, \quad b=X^{\alpha+\alpha^{\prime}}-Y^{\beta} Z^{\gamma^{\prime}} \text { and } c=Y^{\beta+\beta^{\prime}}-X^{\alpha} Z^{\gamma} .
$$

Proposition (2.2) (cf. [6, 12, 19]). Suppose that $M$ has type (I). Then there exists $d_{2} \in \mathfrak{p}^{(2)}$ such that $\mathfrak{p}^{(2)}=\left(d_{2}\right)+\mathfrak{p}^{2}$ and

$$
\begin{aligned}
& X^{\alpha^{\prime}} d_{2}=Z^{\gamma^{\prime}-\gamma} a c-Y^{\beta^{\prime}-\beta} b^{2}, \\
& Y^{\beta} d_{2}=a b-X^{\alpha^{\prime}-\alpha} Z^{\gamma^{\prime}-\gamma} c^{2}, \\
& Z^{r} d_{2}=X^{\alpha^{\prime}-\alpha} Y^{\beta^{\prime}-\beta} b c-a^{2} .
\end{aligned}
$$

We furthermore have

$$
\begin{aligned}
d_{2} & \equiv-Z^{\gamma+2 \gamma^{\prime}} \bmod (X) & & \text { if } \alpha<\alpha^{\prime}, \\
& \equiv-Z^{\gamma+2 \gamma^{\prime}} \bmod (Y) & & \text { if } \beta<\beta^{\prime}, \\
& \equiv-X^{\alpha+2 \alpha^{\prime}} Y^{\beta^{\prime}-\beta} \bmod (Z) & & \text { if } \gamma<\gamma^{\prime} .
\end{aligned}
$$


Proposition (2.3) (cf. [15]). Suppose that $M$ has type (II). Then there exists $d_{2} \in \mathfrak{p}^{(2)}$ such that $\mathfrak{p}^{(2)}=\left(d_{2}\right)+\mathfrak{p}^{2}$ and

$$
\begin{aligned}
& X^{\alpha^{\prime}} d_{2}=Z^{\gamma^{\prime}-\gamma} a c-Y^{\beta^{\prime}-\beta} b^{2}, \\
& Y^{\beta} d_{2}=X^{\alpha-\alpha^{\prime}} a b-Z^{\gamma^{\prime}-\gamma} c^{2}, \\
& Z^{\gamma} d_{2}=Y^{\beta^{\prime}-\beta} b c-X^{\alpha-\alpha^{\prime}} a^{2} .
\end{aligned}
$$

Hence we have $d_{2} \equiv-Y^{\beta+2 \beta^{\prime}} Z^{\gamma^{\prime}-\gamma} \bmod (X)$.

Corollary (2.4). (1) Suppose that $M$ has type (I). Then we have

$$
\begin{aligned}
\mu_{A}\left(\mathfrak{p}^{2)}\right)=4 & \text { if } \beta=\beta^{\prime} \text { or } \gamma=\gamma^{\prime}, \\
=5 & \text { if } \beta<\beta^{\prime} \text { and } \gamma<\gamma^{\prime} .
\end{aligned}
$$

(2) If $M$ has type (II), then $\mu_{A}\left(\mathfrak{p}^{(2)}\right)=7$.

Proof. See [15, (2.3)] for the proof of the assertion (2).

(1) First we assume that $\beta=\beta^{\prime}$. Then by (2.1) $\alpha<\alpha^{\prime}$ or $\gamma<\gamma^{\prime}$. If $\alpha<\alpha^{\prime}$, by (2.2) we have $(X)+\mathfrak{p}^{(2)}=\left(X, Z^{\gamma+2 \gamma^{\prime}}, Y^{2 \beta} Z^{\gamma+\gamma^{\prime}}, Y^{3 \beta} Z^{\gamma^{\prime}}, Y^{4 \beta}\right)$ so that $\mu_{A}\left((X)+\mathfrak{p}^{(2)} /(X)\right)=4$. Hence $\mu_{A}\left(\mathfrak{p}^{(2)}\right)=4$, because

$$
\begin{aligned}
(X)+\mathfrak{p}^{(2)} /(X) & \cong \mathfrak{p}^{(2)} /(X) \cap \mathfrak{p}^{(2)} \\
& =\mathfrak{p}^{(2)} / X \mathfrak{p}^{(2)} .
\end{aligned}
$$

Similarly we get $\mu_{A}\left(\mathfrak{p}^{(2)}\right)=4$ also for the case $\gamma<\gamma^{\prime}$, because $(Z)+\mathfrak{p}^{(2)}=$ $\left(Z, Y^{4 \beta}, X^{\alpha^{\prime}} Y^{3 \beta}, X^{\alpha+\alpha^{\prime}} Y^{2 \beta}, X^{\alpha+2 \alpha^{\prime}}\right)$. A similar argument works to prove the assertion in the case where $\gamma=\gamma^{\prime}$, too.

Next assume that $\beta<\beta^{\prime}$ and $\gamma<\gamma^{\prime}$. Then by (2.2) we have $(Y)+\mathfrak{p}^{(2)}$ $=\left(Y, Z^{\gamma+2 \gamma^{\prime}}, X^{\alpha} Z^{2 \gamma+\gamma^{\prime}}, X^{2 \alpha} Z^{2 \gamma}, X^{2 \alpha+\alpha^{\prime}} Z^{\gamma}, X^{2 \alpha+2 \alpha^{\prime}}\right)$ and so $\mu_{A}\left((Y)+\mathfrak{p}^{(2)} /(Y)\right)=5$. Therefore $\mu_{A}\left(\mathfrak{p}^{(2)}\right)=5$.

\section{$\S 3$. The Cohen-Macaulay property of the $A$-algebra $A\left[\mathfrak{p} t, \mathfrak{p}^{(2)} t^{2}\right]$}

Let us maintain the same notation as in Section 2. The purpose is to prove the following

Theorem (3.1). Suppose that $M$ has type (II) and $\left(\alpha-2 \alpha^{\prime}\right)\left(\beta^{\prime}-2 \beta\right)$ $\left(\gamma^{\prime}-2 \gamma\right) \geq 0$. Then the A-algebra $R=A\left[\mathfrak{p} t, \mathfrak{p}^{(2)} t^{2}\right]$ is a Cohen-Macaulay ring with $r(R)=3$, where $r(R)$ denotes the Cohen-Macaulay type of $R$ (cf. $[8$, p. 4]).

We divide the proof of Theorem (3.1) into several steps. First assume that $M$ has type (II). Let $T_{1}, T_{2}, T_{3}$ and $T_{4}$ be indeterminates over $A$ and 
put $S=A\left[T_{1}, T_{2}, T_{3}, T_{4}\right]$. Let $\psi: S \rightarrow R$ be the homomorphism of $A$-algebras such that $\psi\left(T_{1}\right)=a t, \psi\left(T_{2}\right)=b t, \psi\left(T_{3}\right)=c t$ and $\psi\left(T_{4}\right)=d_{2} t^{2}$, where $d_{2}$ is the element of $\mathfrak{p}^{(2)}$ given in (2.3). Let $P=\operatorname{Ker} \psi$. Then $P$ is a prime ideal in $S$ of $h_{S} P=3$ containing the following five elements

$$
\begin{aligned}
& F_{1}=X^{\alpha} T_{1}+Y^{\beta^{\prime}} T_{2}+Z^{\gamma^{\prime}} T_{3}, \\
& F_{2}=Y^{\beta} T_{1}+Z^{\gamma} T_{2}+X^{\alpha^{\prime}} T_{3}, \\
& G_{1}=X^{\alpha^{\prime}} T_{4}+Y^{\beta^{\prime}-\beta} T_{2}^{2}-Z^{\gamma^{\prime}-\gamma} T_{1} T_{3}, \\
& G_{2}=Y^{\beta} T_{4}+Z^{\gamma^{\prime}-\gamma} T_{3}^{2}-X^{\alpha-\alpha^{\prime}} T_{1} T_{2}, \\
& G_{3}=Z^{\gamma} T_{4}+X^{\alpha-\alpha^{\prime}} T_{1}^{2}-Y^{\beta^{\prime}-\beta} T_{2} T_{3} .
\end{aligned}
$$

We put $J=\left(F_{1}, F_{2}, G_{1}, G_{2}, G_{3}\right) S$. Then $J \subseteq P$ and we have

Lemma (3.2). $S / J$ is a Gorenstein ring of $\operatorname{dim} S / J=4$.

Proof. Because $J$ is generated by the Pfaffians of order 4 in the following 5 by 5 antisymmetric matrix

$$
\left[\begin{array}{rcccc}
0 & T_{4} & T_{3} & -T_{1} & T_{2} \\
-T_{4} & 0 & -X^{\alpha-\alpha^{\prime}} T_{1} & Y^{\beta^{\prime}-\beta} T_{2} & -Z^{\gamma^{\prime}-\gamma} T_{3} \\
-T_{3} & X^{\alpha-\alpha^{\prime}} T_{1} & 0 & Z^{\gamma} & Y^{\beta} \\
T_{1} & -Y^{\beta^{\prime}-\beta} T_{2} & -Z^{\gamma} & 0 & X^{\alpha^{\prime}} \\
-T_{2} & Z^{\gamma^{\prime}-\gamma} T_{3} & -Y^{\beta} & -X^{\alpha^{\prime}} & 0
\end{array}\right],
$$

to see that $S / J$ is a Gorenstein ring of $\operatorname{dim} S / J=4$ it is enough by [2, Theorem 2.1] to show that grade $_{S} J=3$. We put $B=A\left[T_{1}, T_{2}, T_{3}\right]$. Then $F_{1}, F_{2} \in B$ and $R(\mathfrak{p}):=\sum_{n \geq 0} \mathfrak{p}^{n} t^{n} \cong B /\left(F_{1}, F_{2}\right) B$ (cf. [21, Theorem 3.6]). Hence $\left(F_{1}, F_{2}\right) B \in \operatorname{Spec} B$ with $\operatorname{ht}_{B}\left(F_{1}, F_{2}\right) B=2$ and so $\left(F_{1}, F_{2}\right) S \in \operatorname{Spec} S$ with $\mathrm{ht}_{S}\left(F_{1}, F_{2}\right) S=2$. Obviously $G_{1} \notin\left(F_{1}, F_{2}\right) S$. Thus we get that grade $_{S} J \geq 3$, while $\mathrm{ht}_{S} J \leq 3$ as $J \subseteq \mathfrak{n} S$. Hence $\operatorname{grade}_{S} J=3$ as required.

Lemma (3.3). $\quad \operatorname{Ass}_{S} S / J=\{P, \mathfrak{m} S\}$.

Proof (cf. Proof of [9, Theorem 2.1]). Obviously $\operatorname{Ass}_{S} S / J \supseteq\{P, \mathfrak{m} S\}$, so we shall prove the reverse inclusion. Since $\operatorname{Ass}_{S} S / J=\operatorname{Min}_{S} S / J$ by (3.2), we see that $\mathfrak{m} S$ is the unique associated prime ideal of $S / J$ including $\left(X^{\alpha^{\prime}}, Y^{\beta}, Z^{r}\right) A$. Hence there exists $\xi \in\left(X^{\alpha^{\prime}}, Y^{\beta}, Z^{r}\right) A$ such that $\xi \notin Q$ for any $Q \in \operatorname{Ass}_{S} S / J \backslash\{\mathfrak{m} S\}$. We write $\xi=c_{1} X^{\alpha^{\prime}}+c_{2} Y^{\beta}+c_{3} Z^{\gamma}$, where $c_{i} \in A$ for $1 \leq i \leq 3$, and we put $G=c_{1} G_{1}+c_{2} G_{2}+c_{3} G_{3}$. Then $G=\xi T_{4}-\eta$ with $\eta \in A\left[T_{1}, T_{2}, T_{3}\right]$. Let $S^{\prime}:=S[1 / \xi]=A[1 / \xi]\left[T_{1}, T_{2}, T_{3}, T_{4}\right]$ and let $\rho:=\eta / \xi$. Then since $S^{\prime} /\left(F_{1}, F_{2}, T_{4}-\rho\right) \cong A[1 / \xi]\left[T_{1}, T_{2}, T_{3}^{i}\right] /\left(F_{1}, F_{2}\right)$, we get that $\left(F_{1}\right.$, 
$\left.F_{2}, T_{4}-\rho\right) S^{\prime} \in \operatorname{Spec} S^{\prime}$ and $\mathrm{ht}_{S^{\prime}}\left(F_{1}, F_{2}, T_{4}-\rho\right) S^{\prime}=3$ (cf. the proof of (3.2)). Let $Q \in \operatorname{Ass}_{S} S / J \backslash\{\mathfrak{m} S\}$. Then we get $J S^{\prime}=Q S^{\prime}$ because $\left(F_{1}, F_{2}, T_{4}-\rho\right) S^{\prime}$ $\subseteq J S^{\prime} \subseteq Q S^{\prime}$. This means that $P S^{\prime}=Q S^{\prime}$ so that $P=Q$, which completes the proof.

As $J S_{P}=P S_{P}$ by the proof of (3.3), we know that $P$ is the $P$-primary component of $J$. Let $C=S_{\mathrm{m} S}$ and $\mathfrak{a}=J C \cap S$. Then we get

$$
J=P \cap a,
$$

which implies $P=[J: \mathfrak{a}]_{S}$ and $\mathfrak{a}=[J: P]_{S}$ so that we have the following

Lemma (3.4). $P / \mathfrak{d}=[(0): \mathfrak{a} / J]_{S / J}$ and $\mathfrak{a} / J=[(0): P / J]_{S / J}$.

Let $\alpha_{1}=\min \left\{\alpha^{\prime}, \alpha-\alpha^{\prime}\right\}, \quad \beta_{1}=\min \left\{\beta, \beta^{\prime}-\beta\right\}$ and $\gamma_{1}=\left\{\gamma, \gamma^{\prime}-\gamma\right\}$ and put $K=\left(X^{\alpha_{1}}, Y^{\beta_{1}}, Z^{\gamma_{1}}\right) S$.

Proposition (3.5). $\quad R$ is a Cohen-Macaulay ring of $r(R)=3$, if $J C=$ $K C$.

Proof. As $K$ is mS-primary, we have $\mathfrak{a}=J C \cap S=K C \cap S=K$. Hence as $S / K$ is Cohen-Macaulay, we see by (3.4) and [16, Proposition (1.3)] that $R=S / P$ is Cohen-Macaulay, too. Let $K_{R}$ be the canonical module of $R$. Then we get

$$
\begin{aligned}
K_{R} & \cong \operatorname{Hom}_{S / J}(S / P, S / J) \\
& \cong[(0): P / J]_{S / J} \\
& =K / J
\end{aligned}
$$

Therefore we have

$$
\begin{aligned}
r(R) & =\mu_{R}(K / J) \\
& =\ell_{R}(K /(N K+J)) \\
& =\ell_{R}(K / N K) \\
& =3,
\end{aligned}
$$

where $N=\mathfrak{m} R+\left(T_{1}, T_{2}, T_{3}, T_{4}\right) R$.

Now we are ready to prove Theorem (3.1).

Proof of Theorem (3.1).

It is enough to show that $J C=K C$ if $\left(\alpha-2 \alpha^{\prime}\right)\left(\beta^{\prime}-2 \beta\right)\left(\gamma^{\prime}-2 \gamma\right) \geq 0$. Let us respectively denote by $x, y, z$ and $t_{i}$ the images of $X, Y, Z$ and $T_{i}$ in $C / J C$. As $G_{1}, G_{2}$ and $G_{3}$ are in $J C$, we have 


$$
\begin{aligned}
& x^{\alpha^{\prime}} t_{4}+y^{\beta^{\prime}-\beta} t_{2}^{2}-z^{\gamma^{\prime}-\gamma} t_{1} t_{3}=0, \\
& y^{\beta} t_{4}+z^{\gamma^{\prime}-\gamma} t_{3}^{2}-x^{\alpha-\alpha^{\prime}} t_{1} t_{2}=0, \\
& z^{\gamma} t_{4}+x^{\alpha-\alpha^{\prime}} t_{1}^{2}-y^{\beta^{\prime}-\beta} t_{2} t_{3}=0 .
\end{aligned}
$$

By the equations (*1) and (*2) we get

$$
\begin{aligned}
0 & =x^{\alpha^{\prime}} t_{3} t_{4}+y^{\beta^{\prime}-\beta} t_{2}^{2} t_{3}-t_{1}\left(z^{\gamma^{\prime}-\gamma} t_{3}^{2}\right) \\
& =x^{\alpha^{\prime}} t_{3} t_{4}+y^{\beta^{\prime}-\beta} t_{2}^{2} t_{3}-t_{1}\left(x^{\alpha-\alpha^{\prime}} t_{1} t_{2}-y^{\beta} t_{4}\right),
\end{aligned}
$$

so that

$$
x^{\alpha_{1}}\left(x^{\alpha^{\prime}-\alpha_{1}} t_{3} t_{4}-x^{\alpha-\alpha^{\prime}-\alpha_{1}} t_{1}^{2} t_{2}\right)+y^{\beta_{1}}\left(y^{\beta-\beta_{1}} t_{1} t_{4}+y^{\beta^{\prime}-\beta-\beta_{1}} t_{2}^{2} t_{3}\right)=0 .
$$

Suppose that $\gamma^{\prime} \geq 2 \gamma$. Then by $\left({ }^{*} 1\right)$ and $\left({ }^{*} 3\right)$ we have

$$
\begin{aligned}
0 & =x^{\alpha^{\prime}} t_{4}^{2}+y^{\beta^{\prime}-\beta} t_{2}^{2} t_{4}-z^{\gamma^{\prime}-2 \gamma} t_{1} t_{3}\left(z^{\gamma} t_{4}\right) \\
& =x^{\alpha^{\prime}} t_{4}^{2}+y^{\beta^{\prime}-\beta} t_{2}^{2} t_{4}-z^{\gamma^{\prime}-2 \gamma} t_{1} t_{3}\left(y^{\beta^{\prime}-\beta} t_{2} t_{3}-x^{\alpha-\alpha^{\prime}} t_{1}^{2}\right),
\end{aligned}
$$

so that

$$
x^{\alpha_{1}}\left(x^{\alpha^{\prime}-\alpha_{1}} t_{4}^{2}+x^{\alpha-\alpha^{\prime}-\alpha_{1}} z^{\gamma^{\prime}-2 \gamma} t_{1}^{3} t_{3}\right)+y^{\beta_{1}}\left(y^{\beta^{\prime}-\beta-\beta_{1}} t_{2}^{2} t_{4}-y^{\beta^{\prime}-\beta-\beta_{1}} z^{\gamma^{\prime}-2 \gamma} t_{1} t_{2} t_{3}^{2}\right)=0 .
$$

Thus we get

$$
U\left[\begin{array}{l}
x^{\alpha_{1}} \\
y^{\beta_{1}}
\end{array}\right]=\left[\begin{array}{l}
0 \\
0
\end{array}\right]
$$

where

$$
U=\left[\begin{array}{cc}
x^{\alpha^{\prime}-\alpha_{1}} t_{3} t_{4}-x^{\alpha-\alpha^{\prime}-\alpha_{1}} t_{1}^{2} t_{2} & y^{\beta-\beta_{1}} t_{1} t_{4}+y^{\beta^{\prime}-\beta-\beta_{1}} t_{2}^{2} t_{3} \\
x^{\alpha^{\prime}-\alpha_{1}} t_{4}^{2}+x^{\alpha-\alpha^{\prime}-\alpha_{1}} z^{\gamma^{\prime}-2 \gamma} t_{1}^{3} t_{3} & y^{\beta^{\prime}-\beta-\beta_{1}} t_{2}^{2} t_{4}-y^{\beta^{\prime}-\beta-\beta_{1}} z^{\gamma^{\prime}-2 \gamma} t_{1} t_{2} t_{3}^{2}
\end{array}\right] .
$$

Because

$$
\begin{aligned}
\operatorname{det} U= & -x^{\alpha^{\prime}-\alpha_{1}} y^{\beta^{\prime}-\beta-\beta_{1}} z^{\gamma^{\prime}-2 \gamma} t_{1} t_{2} t_{3}^{3} t_{4}-x^{\alpha-\alpha^{\prime}-\alpha_{1}} y^{\beta^{\prime}-\beta-\beta_{1}} t_{1}^{2} t_{2}^{3} t_{4} \\
& -x^{\alpha^{\prime}-\alpha_{1}} y^{\beta-\beta_{1}} t_{1} t_{4}^{3}-x^{\alpha-\alpha^{\prime}-\alpha_{1}} y^{\beta-\beta_{1}} z^{\gamma^{\prime}-2 r} t_{1}^{4} t_{3} t_{4},
\end{aligned}
$$

if $\left(\alpha-2 \alpha^{\prime}\right)\left(\beta^{\prime}-2 \beta\right) \geq 0$ or $\gamma^{\prime}=2 \gamma$, we see that $\operatorname{det} U$ is a unit of $C / J C$ so that $z^{\gamma_{1}}=0$ as well as $x^{\alpha_{1}}=y^{\beta_{1}}=0$, which implies $J C=K C$.

We consider the case where $\gamma^{\prime} \leq 2 \gamma$. Then by $\left({ }^{*} 1\right)$ and $(* 3)$ we get

$$
\begin{aligned}
0 & =z^{2 \gamma-r^{\prime}} t_{4}\left(z^{\gamma^{\prime}-\gamma} t_{1} t_{3}\right)+x^{\alpha-\alpha^{\prime}} t_{1}^{3} t_{3}-y^{\beta^{\prime}-\beta} t_{1} t_{2} t_{3}^{2} \\
& =z^{2 \gamma-r^{\prime}} t_{4}\left(x^{\alpha^{\prime}} t_{4}+y^{\beta^{\prime}-\beta} t_{2}^{2}\right)+x^{\alpha-\alpha^{\prime}} t_{1}^{3} t_{3}-y^{\beta^{\prime}-\beta} t_{1} t_{2} t_{3}^{2},
\end{aligned}
$$

so that

$$
x^{\alpha_{1}}\left(x^{\alpha^{\prime}-\alpha_{1}} z^{2 \gamma-\gamma^{\prime}} t_{4}^{2}+x^{\alpha-\alpha^{\prime}-\alpha_{1}} t_{1}^{3} t_{3}\right)+y^{\beta_{1}}\left(y^{\beta^{\prime}-\beta-\beta_{1}} z^{2 \gamma-\gamma^{\prime}} t_{2}^{2} t_{4}-y^{\beta^{\prime}-\beta-\beta_{1}} t_{1} t_{2} t_{3}^{2}\right)=0 .
$$


Hence we have

$$
U\left[\begin{array}{l}
x^{\alpha_{1}} \\
y^{\beta_{1}}
\end{array}\right]=\left[\begin{array}{l}
0 \\
0
\end{array}\right]
$$

where

$$
U=\left[\begin{array}{cc}
x^{\alpha^{\prime}-\alpha_{1}} t_{3} t_{4}-x^{\alpha-\alpha^{\prime}-\alpha_{1}} t_{1}^{2} t_{2} & y^{\beta-\beta_{1}} t_{1} t_{4}+y^{\beta^{\prime}-\beta-\beta_{1}} t_{2}^{2} t_{3} \\
x^{\alpha^{\prime}-\alpha_{1}} z^{2 \gamma-\gamma^{\prime}} t_{4}^{2}+x^{\alpha-\alpha^{\prime}-\alpha_{1}} t_{1}^{3} t_{3} & y^{\beta^{\prime}-\beta-\beta_{1}} z^{2 \gamma-\gamma^{\prime}} t_{2}^{2} t_{4}-y^{\beta^{\prime}-\beta-\beta_{1}} t_{1} t_{2} t_{3}^{2}
\end{array}\right] .
$$

If $\left(\alpha-2 \alpha^{\prime}\right)\left(\beta^{\prime}-2 \beta\right) \leq 0$ or $\gamma^{\prime}=2 \gamma$, we see that $\operatorname{det} U$ is a unit in $C(J C$ whence $x^{\alpha_{1}}=y^{\beta_{1}}=z^{\gamma_{1}}=0$. Thus we have $J C=K C$ in this case, too. This together with the result in the case where $\gamma^{\prime} \geq 2 \gamma$ completes the proof of Theorem (3.1).

Corollary (3.6). Suppose that $M$ has type (II) and satisfies one of the following conditions

(1) $\alpha^{\prime}=\beta=\gamma=1$.

(2) $\alpha=2 \alpha^{\prime}, \beta^{\prime}=2 \beta$ or $\gamma^{\prime}=2 \gamma$.

Then $R=A\left[\mathfrak{p} t, \mathfrak{p}^{(2)} t^{2}\right]$ is a Cohen-Macaulay ring of $r(R)=3$.

Example (3.7). $\quad R=A\left[\mathfrak{p} t, \mathfrak{p}^{(2)} t^{2}\right]$ is a Cohen-Macaulay ring of $r(R)=3$ for the following prime ideals $\mathfrak{p}$ :

(1) $\mathfrak{p}=\mathfrak{p}\left(n^{2}+2 n+2, n^{2}+2 n+1, n^{2}+n+1\right)$, where $2 \leq n \in Z$.

(2) $\mathfrak{p}=\mathfrak{p}\left(n^{2}, n^{2}+1, n^{2}+n+1\right)$, where $3 \leq n \in Z$.

(3) $\mathfrak{p}=\mathfrak{p}(17,10,19)$.

Proof. The prime ideals $\mathfrak{p}$ of (1), (2) and (3) are respectively generated by the maximal minors of the inatrix

$$
\left[\begin{array}{ccc}
X^{n} & Y^{n} & Z^{n+1} \\
Y & Z & X
\end{array}\right], \quad\left[\begin{array}{ccc}
X^{n} & Y^{n} & Z^{n-1} \\
Y & Z & X
\end{array}\right] \text { and }\left[\begin{array}{ccc}
X^{3} & Y^{4} & Z^{2} \\
Y^{3} & Z & X
\end{array}\right]
$$

Hence we see by (3.6) that $R$ is a Cohen-Macaulay ring of $r(R)=3$.

Example (3.8). Let $\mathfrak{p}=\mathfrak{p}(13,14,17)$. Then the $A$-algebra $R=A[p t$, $\left.\mathfrak{p}^{(2)} t^{2}\right]$ is not a Cohen-Macaulay ring.

Proof. Notice that $\mathfrak{p}$ is generated by the maximal minors of the matrix

$$
M=\left[\begin{array}{ccc}
X^{3} & Y^{3} & Z^{3} \\
Y & Z & X^{2}
\end{array}\right]
$$

of type (II). We put $a=Z^{4}-X^{2} Y^{3}, b=X^{5}-Y Z^{3}$ and $c=Y^{4}-X^{3} Z$. Then by (2.3) there exists $d_{2} \in \mathfrak{p}^{(2)}$ such that 


$$
\begin{aligned}
& X^{2} d_{2}=Y^{2} b^{2}-Z^{2} a c, \\
& Y d_{2}=Z^{2} c^{2}-X a b \text { and } \\
& Z d_{2}=X a^{2}-Y^{2} b c .
\end{aligned}
$$

Let $S=A\left[T_{1}, T_{2}, T_{3}, T_{4}\right]$ and $P$ be as in the proof of (3.1). Then the following elements

$$
\begin{aligned}
& F_{1}=X^{3} T_{1}+Y^{3} T_{2}+Z^{3} T_{3}, \\
& F_{2}=Y T_{1}+Z T_{2}+X^{2} T_{3}, \\
& G_{1}=X^{2} T_{4}-Y^{2} T_{2}^{2}+Z^{2} T_{1} T_{3}, \\
& G_{2}=Y T_{4}-Z^{2} T_{3}^{2}+X T_{1} T_{2} \text { and } \\
& G_{3}=Z T_{4}-X T_{1}^{2}+Y^{2} T_{2} T_{3}
\end{aligned}
$$

are in $P$. We put $J=\left(F_{1}, F_{2}, G_{1}, G_{2}, G_{3}\right) S, Q=\mathfrak{m} S, C=S_{Q}$ and $\mathfrak{a}=J C \cap S$. As is shown in the proof of (3.1), we have $J=P \cap a$. Therefore by (3.4) and [16, Proposition 1.3] it is enough to show that $S / \mathfrak{a}$ is not CohenMacaulay. First we prove

Claim 1. $Q^{2} C \subseteq J C$.

Proof of Claim 1. We respectively denote by $x, y, z$ and $t_{i}$ the images of $X, Y, Z$ and $T_{i}$ in $C / J C$. It suffices to show that $(x, y, z)^{2}=(0)$. Since $G_{1}, G_{2}$ and $G_{3}$ are in $J$, we have

(*1) $x^{2} t_{4}-y^{2} t_{2}^{2}+z^{2} t_{1} t_{3}=0$

(*2) $y t_{4}-z^{2} t_{3}^{2}+x t_{1} t_{2}=0$,

(*3) $z t_{4}-x t_{1}^{2}+y^{2} t_{2} t_{3}=0$.

By the equations (*1) and (*2) we get $x^{2} t_{3} t_{4}-y^{2} t_{2}^{2} t_{3}+y t_{1} t_{4}+x t_{1}^{2} t_{2}=0$ and so $y=u x$, where $u=\left(t_{1}^{2} t_{2}+x t_{3} t_{4}\right) /\left(y t_{2}^{2} t_{3}-t_{1} t_{4}\right)$. Then by $(* 3)$ we have $z t_{4}-x t_{1}^{2}+u^{2} x^{2} t_{2} t_{3}=0$ so that $z=v x$ with $v=\left(t_{1}^{2}-u^{2} x t_{2} t_{3}\right) / t_{4}$. Thus the equation $\left({ }^{*} 1\right)$ yields $x^{2}\left(t_{4}-u^{2} t_{2}^{2}+v^{2} t_{1} t_{3}\right)=0$. Let $Q^{\prime}=Q C / J C$. Then as $u \equiv-t_{1} t_{2} / t_{4} \bmod Q^{\prime}$ and as $v \equiv t_{1}^{2} / t_{4} \bmod Q^{\prime}$, we get $t_{4}-u^{2} t_{2}^{2}+v^{2} t_{1} t_{3} \equiv\left(t_{4}^{3}-\right.$ $\left.t_{1}^{2} t_{4}^{2}+t_{1}^{5} t_{3}\right) / t_{4}^{2} \bmod Q^{\prime}$. Hence $t_{4}-u^{2} t_{2}^{2}+v^{2} t_{1} t_{3}$ is a unit in $C / J C$ and so $x^{2}=0$, which claims that $(x, y, z)^{2}=0$, because $y=u x$ and $z=v x$.

We put $I=(f, g, h) S+Q^{2}$, where $f=Y T_{4}+X T_{1} T_{2}, g=Z T_{4}-X T_{1}^{2}$ and $h=Y T_{1}+Z T_{2}$. Then $I C=J C$ by Claim 1 . We want to show that $I$ is $Q$-primary and $S / I$ is not Cohen-Macaulay, which will complete the proof of (3.8) because $I=\mathfrak{a}$.

Let us consider the exact sequence

$$
0 \longrightarrow Q / I \longrightarrow S / I \longrightarrow S / Q \longrightarrow 0
$$


CLAIM 2. $\operatorname{rank}_{S / Q} Q / I=1$.

Proof of Claim 2. We shall show that $\ell_{C}(Q C / I C)=1$. Notice that $\ell_{C}(Q C / I C) \leq 1$, because $Q C \supseteq I C \supseteq Q^{2} C$ and $Q C=(X, f, g) C$. Hence it is enough to show that $Q C \neq I C$. Assume the contrary. Then $Q C=(f, g, h) C$ by Nakayama's lemma, which is impossible because

$$
\left[\begin{array}{l}
f \\
g \\
h
\end{array}\right]=U\left[\begin{array}{l}
X \\
Y \\
Z
\end{array}\right] \text { where } U=\left[\begin{array}{ccc}
T_{1} T_{2} & T_{4} & 0 \\
-T_{1}^{2} & 0 & T_{4} \\
0 & T_{1} & T_{2}
\end{array}\right]
$$

and because $\operatorname{det} U=0$.

We consider the following complex

$$
\begin{aligned}
0 \longrightarrow S / Q \stackrel{\varphi_{3}}{\longrightarrow}(S / Q)^{3} \stackrel{\varphi_{2}}{\longrightarrow} \quad(S / Q)^{2} \stackrel{\varphi_{1}}{\longrightarrow} S / Q, \\
{\left[\begin{array}{r}
T_{2} \\
T_{1} \\
-T_{4}
\end{array}\right] \quad\left[\begin{array}{ccc}
-T_{1}^{2} & T_{1} T_{2} & 0 \\
0 & T_{4} & T_{1} \\
T_{4} & 0 & T_{2}
\end{array}\right] \quad\left[\begin{array}{lllll}
T_{4} & -T_{1} T_{2} & T_{1}^{2}
\end{array}\right] }
\end{aligned}
$$

which is exact by [1, Theorem]. Let $\varepsilon:(S / Q)^{3} \rightarrow Q / I$ be the $S / Q$-homomorphism such that $\varepsilon\left(e_{1}\right)=X \bmod I, \varepsilon\left(e_{2}\right)=Y \bmod I$ and $\varepsilon\left(e_{3}\right)=Z \bmod I$, where $e_{1}, e_{2}$ and $e_{3}$ are the standard basis of $(S / Q)^{3}$. Then since $\varepsilon \circ \varphi_{2}=0$, there exists an epimorphism $\rho:\left(T_{4},-T_{1} T_{2}, T_{1}^{2}\right)(S / Q) \rightarrow Q / I$ such that $\rho \circ \varphi_{1}=\varepsilon$. Then $\rho$ is an isomorphism by Claim 2. Therefore we have that $Q / I$ is embedded, via $\rho$, in $S / Q$ and that proj. $\operatorname{dim}_{S / Q} Q / I=2$. Thus we see that $\operatorname{Ass}_{S} Q ! I=\{Q\}$ and $\operatorname{depth}_{S_{N}}(Q / I)_{N}=2$, where $N=\mathfrak{m} S+\left(T_{1}, T_{2}, T_{3}, T_{4}\right) S$ (notice that $S / Q$ is a polynomial ring with four variables over the field $k=A / \mathfrak{m})$. Hence by the exact sequence (\#) we get $\operatorname{Ass}_{S} S / I=\{Q\}$ and $\operatorname{depth}_{S_{N}}(S / I)_{N}=2$, so that $S / I$ is not a Cohen-Macaulay ring because $\operatorname{dim}(S / I)_{N}=4$. This completes the proof of (3.8).

\section{$\S 4$. The generation of $R_{s}(\mathfrak{p})$ in degree two}

Let $A$ be a regular local ring of $\operatorname{dim} A=3$ and $\mathfrak{p}$ a prime ideal in $A$ of $\operatorname{dim} A / \mathfrak{p}=1$. In this general situation we shall characterize prime ideals $\mathfrak{p}$ whose symbolic Rees algebras $R_{s}(\mathfrak{p})$ are generated by $\mathfrak{p} t$ and $\mathfrak{p}^{(2)} t^{2}$. The first result is

Theorem (4.1). Suppose that $A / \mathrm{m}$ is infinite. Then the following conditions are equivalent. 
(1) $R_{s}(\mathfrak{p})=A\left[\mathfrak{p} t, \mathfrak{p}^{(2)} t^{2}\right]$.

(2) $\ell_{A}(A /(f, g, x))=4 \cdot \ell_{A}(A /(x)+\mathfrak{p})$ for some $f, g \in \mathfrak{p}^{(2)}$ and $x \in \mathfrak{m} \backslash \mathfrak{p}$.

(3) $(f, g): \mathfrak{p}^{(2)}=\mathfrak{p}$ for some $f, g \in \mathfrak{p}^{(2)}$.

(4) $(f, g) \supseteq \mathfrak{p}^{(3)}$ for some $f, g \in \mathfrak{p}^{(2)}$.

When this is the case, $R_{s}(\mathfrak{p})$ is a Gorenstein ring.

Proof. See [5, Corollaries (3.8) and (3.9)] for the implication (2) $\Rightarrow(1)$ and the last assertion.

$(1) \Rightarrow(2) \quad$ As $\mathfrak{p}^{(n)}=\mathfrak{p p}^{(n-1)}+\mathfrak{p}^{(2)} \mathfrak{p}^{(n-2)}(n \geq 1)$, we get $\mathfrak{p}^{(2 n)}=\left[\mathfrak{p}^{(2)}\right]^{n}$ and $\mathfrak{p}^{(2 n+1)}=\mathfrak{p}\left[\mathfrak{p}^{(2)}\right]^{n}$ for all $n \geq 1$. Hence $\operatorname{depth} A /\left[\mathfrak{p}^{(2)}\right]^{n}=1$ for any $n \geq 1$ and so by [3, Corollary, (i)] we may choose $f, g \in \mathfrak{p}^{(2)}$ so that $\left[\mathfrak{p}^{(2)}\right]^{r+1}=(f, g)\left[\mathfrak{p}^{(2)}\right]^{r}$ for some $r \geq 0$. Therefore we get the condition (2) by the proof of [11, Theorem 3.1].

$(2) \Rightarrow(4) \quad$ See [5, Proposition (3.4)].

(4) $\Rightarrow$ (2) Let $B=A_{\mathfrak{p}}$ and $\mathfrak{n}=\mathfrak{p} A_{\mathfrak{p}}$. Then $\mathfrak{n}^{2} \neq(f, g) B \supseteq \mathfrak{n}^{3}$ (recall that $\left.\mu_{A}\left(\mathfrak{n}^{2}\right)=3\right)$. Therefore we get $\ell_{A}\left(\mathfrak{n}^{2} /(f, g) B\right)=1$ as $B /(f, g) B$ is a Gorenstein ring. Consequently $\ell_{B}(B /(f, g) B)=4$ and so by the additive formula of multiplicity (cf. [18, p. 126]) we have

$$
\begin{aligned}
\ell_{A}(A /(f, g, x)) & =e_{x A}(A /(f, g)) \\
& =\ell_{B}(B /(f, g) B) \cdot e_{x A}(A / \mathfrak{p}) \\
& =4 \cdot \ell_{A}(A /(x)+\mathfrak{p})
\end{aligned}
$$

for any $x \in \mathfrak{m} \backslash \mathfrak{p}$. Thus we get the condition (2).

$(4) \Rightarrow(3) \quad$ Notice that $(f, g)$ is a $\mathfrak{p}$-primary ideal and $\mathfrak{p}^{(2)} \not \subset(f, g)$. Then we have $(f, g): \mathfrak{p}^{(2)} \subseteq \mathfrak{p}$ so that $(f, g): \mathfrak{p}^{(2)}=\mathfrak{p}$, because $(f, g): \mathfrak{p}^{(2)} \supseteq \mathfrak{p}$ by the assumption (4).

$(3) \Rightarrow(4) \quad$ As $\mathfrak{p}^{(2)} \supseteq(f, g) \supseteq \mathfrak{p} \mathfrak{p}^{(2)}$, we have that $(f, g) \supseteq \mathfrak{p}^{3}$ and $\mathfrak{p}=\sqrt{(f, g)}$. Hence $(f, g) \supseteq \mathfrak{p}^{(3)}$, because $(f, g)$ is a $\mathfrak{p}$-primary ideal of $A$.

For prime ideals $\mathfrak{p}$ with $\mu_{A}\left(\mathfrak{p}^{(2)} / \mathfrak{p}^{2}\right) \leq 1$, we can improve Theorem (4.1) as follows.

TheOREM (4.2). Suppose that $A / \mathfrak{m}$ is infinite. Then the following conditions are equivalent.

(1) $R_{s}(\mathfrak{p})=A\left[\mathfrak{p} t, \mathfrak{p}^{(2)} t^{2}\right]$ and $\mu_{A}\left(\mathfrak{p}^{(2)} / \mathfrak{p}^{2}\right) \leq 1$.

(2) $\ell_{A}(A /(f, g, x))=2 \cdot \ell_{A}(A /(x)+\mathfrak{p})$ for some $f \in \mathfrak{p}, g \in \mathfrak{p}^{(2)}$ and $x \in \mathfrak{m} \backslash \mathfrak{p}$.

(3) $\mathfrak{p}^{(2)}=f \mathfrak{p}+(g)$ for some $f \in \mathfrak{p}$ and $g \in \mathfrak{p}^{(2)}$.

(4) $(f, g): \mathfrak{p}=\mathfrak{p}$ for some $f \in \mathfrak{p}$ and $g \in \mathfrak{p}^{(2)}$.

When this is the case, $R_{s}(p)$ is a Gorenstein ring. 
Proof. $\quad(1) \Rightarrow(2) \quad$ By (4.1) we can choose $\alpha, \beta \in \mathfrak{p}^{(2)}$ so that $\ell_{A}(A /(\alpha, \beta, x))$ $=4 \cdot \ell_{A}(A /(x)+\mathfrak{p})$ for some $x \in \mathfrak{m} \backslash \mathfrak{p}$. Then $\mathfrak{p}^{(3)}=(\alpha, \beta) \mathfrak{p}$ by [5, Proposition (3.4), Lemma (3.5)]. If $(\alpha, \beta) \subseteq \mathfrak{m} \mathfrak{p}^{(2)}+\mathfrak{p}^{2}$, we have $\mathfrak{p}^{(3)} \subseteq \mathfrak{m} \mathfrak{p}^{(3)}+\mathfrak{p}^{3}$ so that $\mathfrak{p}^{(3)}=\mathfrak{p}^{3}$ by Nakayama's lemma. Then as $\mu_{A}(\mathfrak{p})=2$ by [12, (2.5) Corollary], the assertion (2) is clear. Therefore we may assume $\beta \notin \mathfrak{m} \mathfrak{p}^{(2)}+\mathfrak{p}^{2}$. Let $g=\beta$. Then we have $\mathfrak{p}^{(2)}=(g)+\mathfrak{p}^{2}$ as $\mu_{A}\left(\mathfrak{p}^{(2)} / \mathfrak{p}^{2}\right) \leq 1$ by our assumption. We put $B=A / g A$ and $P=\mathfrak{p} / g A$. Then as $P^{2}=\mathfrak{p}^{(2)} / g A$, we have that $P^{2}$ is $P$-primary. Because $\left[\mathfrak{p}^{(2)}\right]^{2}=(\alpha, g) \mathfrak{p}^{(2)}$ (cf. The proof of [11, Theorem 3.1]), we get $P^{4}=\alpha P^{2}$ whence $\lambda(P)=1$. Choose $f \in \mathfrak{p}$ so that $P^{r+1}=f P^{r}$ for some $r \geq 0$. Then as $v\left(B_{P}\right)=2=e\left(B_{P}\right)$ (notice that $g \in \mathfrak{p}^{(2)} \backslash \mathfrak{p}^{(3)}$, cf. [5, Proposition (3.7), (3)]), we see $P^{2} B_{P}=f P B_{P}$ by [17, 3.8. Theorem] so that $P^{2}=f P$, because $P^{2}$ and $f P$ are $P$-primary ideals of $B$. Hence $\mathfrak{p}^{(2)} \subseteq$ $(f, g) \subseteq \mathfrak{p}$ and we have $\mathfrak{p}^{(2)}+(f)=(f, g)$. Thus $(f, g)$ is a $\mathfrak{p}$-primary ideal and $f \notin \mathfrak{p}^{(2)}$, whence

$$
\begin{aligned}
\ell_{A}(A /(f, g, x))= & e_{x A}\left(A /(f)+\mathfrak{p}^{(2)}\right) \\
& =\ell_{A_{\mathfrak{p}}}\left(A_{\mathfrak{p}} / f A_{\mathfrak{p}}+\mathfrak{p}^{2} A_{\mathfrak{p}}\right) \cdot e_{x A}(A / \mathfrak{p}) \\
& =2 \cdot \ell_{A}(A /(x)+\mathfrak{p})
\end{aligned}
$$

for any $x \in \mathfrak{m} \backslash \mathfrak{p}$.

$(2) \Rightarrow(1)$ See [5, Propositions (3.4) and (3.7)].

$(2) \Rightarrow(3) \quad$ As $\mathfrak{p}^{(2)} \subseteq(f, g)$ by [5, Proposition (3.4)], we get $\mathfrak{p}^{(2)}=f \mathfrak{p}+(g)$ by $[5$, Lemma (3.5)].

$(3) \Rightarrow(4) \quad$ As $(f, g) \supseteq \mathfrak{p}^{(2)},(f, g)$ is $\mathfrak{p}$-primary and $(f, g): \mathfrak{p} \supseteq \mathfrak{p}$. Therefore $(f, g): \mathfrak{p}=\mathfrak{p}$, because $\mathfrak{p} \neq(f, g)$.

(4) $\Rightarrow(2) \quad$ As $\mathfrak{p}^{2} \subseteq(f, g) \subseteq \mathfrak{p}$, we see that $(f, g)$ is $\mathfrak{p}$-primary. Therefore $\mathfrak{p}^{(2)} \subseteq(f, g)$ so that $\mathfrak{p}^{(2)}+(f)=(f, g)$, which implies $f \notin \mathfrak{p}^{(2)}$. Hence we have $\ell_{A_{\mathfrak{p}}}\left(A_{\mathfrak{p}} / f A_{\mathfrak{p}}+\mathfrak{p}^{2} A_{\mathfrak{p}}\right)=2$ and so $\ell_{A}(A /(f, g, x))=2 \cdot \ell_{A}(A /(x)+\mathfrak{p})$ for any $x \in \mathfrak{m} \backslash \mathfrak{p}$. Thus we get the assertion (2).

In the rest of this section let $A, \mathfrak{p}$ and $M$ be as in Section 2. Then by (4.2) we get the following

Corollary (4.3) (cf. [9, Corollary 2.12]). The following conditions are equivalent.

(1) $R_{s}(\mathfrak{p})=A\left[\mathfrak{p} t, \mathfrak{p}^{(2)} t^{2}\right]$.

(2) $M$ has type (I) and (i) $\beta=\beta^{\prime}$ or (ii) $\alpha=\alpha^{\prime}$ and $\gamma=\gamma^{\prime}$.

When this is the case, $R_{s}(\mathfrak{p})$ is a Gorenstein ring.

Proof. $\quad(1) \Rightarrow(2)$ Since $\mu_{A}\left(\mathfrak{p}^{(2)} / \mathfrak{p}^{2}\right) \leq 1$ by $(2.2)$ and (2.3), we get $\mu_{A}\left(\mathfrak{p}^{(2)}\right)$ 
$\leq 4$ by (3) of (4.2) so that $M$ has type (I) and $\beta=\beta^{\prime}$ or $\gamma=\gamma^{\prime}$ by (2.4). Suppose that $\beta<\beta^{\prime}$ and $\gamma=\gamma^{\prime}$. We shall prove that $\alpha=\alpha^{\prime}$. Since we get

$$
\begin{aligned}
& (Y)+\mathfrak{p p}^{(2)}=(Y)+\left(Z^{5 \gamma}, X^{\alpha} Z^{4 \gamma}, X^{3 \alpha} Z^{3 \gamma}, X^{3 \alpha+\alpha^{\prime}} Z^{2 \gamma}, X^{3 \alpha+2 \alpha^{\prime}} Z^{\gamma}, X^{3 \alpha+3 \alpha^{\prime}}\right) \\
& \text { if } 2 \alpha<\alpha^{\prime}, \\
& =(Y)+\left(Z^{5 \gamma}, X^{\alpha} Z^{4 \gamma}, X^{\alpha+\alpha^{\prime}} Z^{3 \gamma}, X^{3 \alpha+\alpha^{\prime}} Z^{2 \gamma}, X^{3 \alpha+2 \alpha^{\prime}} Z^{\gamma}, X^{3 \alpha+3 \alpha^{\prime}}\right) \\
& \text { if } 2 \alpha \geq \alpha^{\prime}
\end{aligned}
$$

by (2.2), we obtain

$$
\begin{aligned}
\ell_{A}\left(A /(Y)+\mathfrak{p p}^{(2)}\right) & =13 \alpha \gamma+6 \alpha^{\prime} \gamma & & \text { if } 2 \alpha<\alpha^{\prime}, \\
& =11 \alpha \gamma+7 \alpha^{\prime} \gamma & & \text { if } 2 \alpha \geq \alpha^{\prime} .
\end{aligned}
$$

On the other hand the condition (1) implies $\mathfrak{p p}^{(2)}=\mathfrak{p}^{(3)}$ so that

$$
\begin{aligned}
\ell_{A}\left(A /(Y)+\mathfrak{p p}^{(2)}\right) & =\ell_{A}\left(A /(Y)+\mathfrak{p}^{(3)}\right) \\
& =e_{Y A}\left(A / \mathfrak{p}^{(3)}\right) \\
& =\ell_{A_{\mathfrak{p}}}\left(A_{\mathfrak{p}} / \mathfrak{p}^{3} A_{\mathfrak{p}}\right) \cdot \ell_{A}(A /(Y)+\mathfrak{p}) \\
& =6\left(2 \alpha \gamma+\alpha^{\prime} \gamma\right) .
\end{aligned}
$$

Thus we have

$$
\begin{aligned}
12 \alpha \gamma+6 \alpha^{\prime} \gamma & =13 \alpha \gamma+6 \alpha^{\prime} \gamma & & \text { if } 2 \alpha<\alpha^{\prime}, \\
& =11 \alpha \gamma+7 \alpha^{\prime} \gamma & & \text { if } 2 \alpha \geq \alpha^{\prime} .
\end{aligned}
$$

Hence $2 \alpha \geq \alpha^{\prime}$ and $\alpha \gamma=\alpha^{\prime} \gamma$ so that $\alpha=\alpha^{\prime}$, as required.

(2) $\Rightarrow$ (1) First we assume $\beta=\beta^{\prime}$. Then $\alpha<\alpha^{\prime}$ or $\gamma<\gamma^{\prime}$ by (2.1). Let $d_{2}$ be the element obtained by (2.2). If $\alpha<\alpha^{\prime}$ (resp. $\gamma<\gamma^{\prime}$ ), then $d_{2} \equiv$ $-Z^{\gamma+2 \gamma^{\prime}} \bmod (X)\left(\right.$ resp. $\left.d_{2} \equiv-X^{\alpha+2 \alpha^{\prime}} \bmod (Z)\right)$ so that

$$
\begin{aligned}
& \qquad \begin{aligned}
\ell_{A}\left(A /\left(c, d_{2}, X\right)\right) & =\ell_{A}\left(A /\left(Y^{2 \beta}, Z^{\gamma+2 \gamma^{\prime}}, X\right)\right) \\
& =2 \beta \gamma+4 \beta \gamma^{\prime} \\
& =2 \cdot \ell_{A}(A /(X)+\mathfrak{p})
\end{aligned} \\
& \text { (resp. } \ell_{A}\left(A /\left(c, d_{2}, Z\right)\right)=\ell_{A}\left(A /\left(Y^{2 \beta}, X^{\alpha+2 \alpha^{\prime}}, Z\right)\right) \\
& =2 \alpha \beta+4 \alpha^{\prime} \beta \\
& \left.=2 \cdot \ell_{A}(A /(Z)+\mathfrak{p})\right),
\end{aligned}
$$

whence $R_{s}(\mathfrak{p})=A\left[\mathfrak{p} t, \mathfrak{p}^{(2)} t^{2}\right]$ by (4.2).

Next assume that $\alpha=\alpha^{\prime}$ and $\gamma=\gamma^{\prime}$. Then $\beta<\beta^{\prime}$ by (2.1). Hence by (2.2) we get $d_{2} \equiv-Z^{3 \gamma} \bmod (Y)$ so that 


$$
\begin{aligned}
\ell_{A}\left(A /\left(b, d_{2}, Y\right)\right) & =\ell_{A}\left(A /\left(X^{2 \alpha}, Z^{3 \gamma}, Y\right)\right) \\
& =6 \alpha \gamma \\
& =2 \cdot \ell_{A}(A /(Y)+\mathfrak{p})
\end{aligned}
$$

Thus $R_{s}(\mathfrak{p})=A\left[\mathfrak{p} t, \mathfrak{p}^{(2)} t^{2}\right]$ by (4.2), which completes the proof of (4.3).

\section{§5. The third symbolic power $\mathfrak{p}^{(3)}$}

The purpose of this section is to investigate the structure of $\mathfrak{p}^{(3)}$ for the prime ideals $\mathfrak{p}$ generated by the maximal minors of matrices $M$ of the form

$$
M=\left[\begin{array}{ccc}
X^{\alpha} & Y^{\beta^{\prime}} & Z^{\gamma^{\prime}} \\
Y^{\beta} & Z^{\gamma} & X^{\alpha^{\prime}}
\end{array}\right]
$$

where $X, Y, Z$ is a regular system of parameters for $A$ and $\alpha, \beta, \gamma, \alpha^{\prime}, \beta^{\prime}, \gamma^{\prime}$ are positive integers. We put $a=Z^{\gamma+\gamma^{\prime}}-X^{\alpha^{\prime}} Y^{\beta^{\prime}}, b=X^{\alpha+\alpha^{\prime}}-Y^{\beta} Z^{\gamma^{\prime}}$ and $c=Y^{\beta+\beta^{\prime}}-X^{\alpha} Z^{\gamma}$.

We begin with the case where $M$ has type (I). By the equation $Y^{\beta} a+Z^{\gamma} b+X^{\alpha^{\prime}} c=0$ and those given in (2.2), we have

$$
\begin{array}{ll}
b\left(X^{\alpha} d_{2}+Y^{\beta^{\prime}-\beta} b^{2}\right)=Z^{\gamma^{\prime}-\gamma} a b c=Z^{\gamma^{\prime}-\gamma} c\left(Y^{\beta} d_{2}+X^{\alpha^{\prime}-\alpha} Z^{\gamma^{\prime}-\gamma} c^{2}\right), \\
a c\left(-Y^{\beta} a-X^{\alpha^{\prime}} c\right)=Z^{\gamma} a b c=Z^{2 \gamma-\gamma^{\prime}} b\left(X^{\alpha} d_{2}+Y^{\beta^{\prime}-\beta} b^{2}\right) & \text { if } 2 \gamma \geq \gamma^{\prime}, \\
Z^{\gamma^{\prime}-2 \gamma} a c\left(-Y^{\beta} a-X^{\alpha^{\prime}} c\right)=Z^{\gamma^{\prime}-\gamma} a b c=b\left(X^{\alpha} d_{2}+Y^{\beta^{\prime}-\beta} b^{2}\right) & \text { if } 2 \gamma<\gamma^{\prime}
\end{array}
$$

so that

$$
\begin{array}{ll}
X^{\alpha} b d_{2}-X^{\alpha^{\prime}-\alpha} Z^{2 \gamma^{\prime}-2 \gamma} c^{3}=Y^{\beta} Z^{\gamma^{\prime}-\gamma} c d_{2}-Y^{\beta^{\prime}-\beta} b^{3}, & \\
X^{\alpha} Z^{2 \gamma-\gamma^{\prime}} b d_{2}+X^{\alpha^{\prime}} a c^{2}=-Y^{\beta} a^{2} c-Y^{\beta^{\prime}-\beta} Z^{2 \gamma-\gamma^{\prime}} b^{3} & \text { if } 2 \gamma \geq \gamma^{\prime}, \\
X^{\alpha} b d_{2}+X^{\alpha^{\prime}} Z^{\gamma^{\prime}-2 \gamma} a c^{2}=-Y^{\beta} Z^{\gamma^{\prime}-2 \gamma} a^{2} c-Y^{\beta^{\prime}-\beta} b^{3} & \text { if } 2 \gamma<\gamma^{\prime} .
\end{array}
$$

Theorem (5.4). Suppose that $M$ has type (I).

(1) Assume $2 \alpha<\alpha^{\prime}$ or $2 \gamma<\gamma^{\prime}$. Then there exists $d_{3} \in \mathfrak{p}^{(3)}$ such that $\mathfrak{p}^{(3)}=\left(d_{3}\right)+\mathfrak{p p}^{(2)}$ and

$$
\begin{array}{ll}
d_{3} \equiv Z^{\gamma+3 \gamma^{\prime}} \bmod (X) & \text { if } 2 \alpha<\alpha^{\prime} \text { and } 2 \beta<\beta^{\prime}, \\
d_{3} \equiv Y^{2 \beta-\beta^{\prime}} Z^{\gamma+3 \gamma^{\prime}} \bmod (X) & \text { if } 2 \alpha<\alpha^{\prime} \text { and } 2 \beta \geq \beta^{\prime}, \\
d_{3} \equiv-X^{2 \alpha+3 \alpha^{\prime}} Y^{\beta^{\prime}-2 \beta} \bmod (Z) & \text { if } 2 \beta<\beta^{\prime} \text { and } 2 \gamma<\gamma^{\prime}, \\
d_{3} \equiv-X^{2 \alpha+3 \alpha^{\prime}} \bmod (Z) & \text { if } 2 \beta \geq \beta^{\prime} \text { and } 2 \gamma<\gamma^{\prime} .
\end{array}
$$

(2) Assume $2 \alpha \geq \alpha^{\prime}$ and $2 \gamma \geq \gamma^{\prime}$, Then there exist $d_{3} \in \mathfrak{p}^{(3)}$ and $d_{3}^{\prime} \in \mathfrak{p}^{(31}$ such that $\mathfrak{p}^{(3)}=\left(d_{3}, d_{3}^{\prime}\right)+\mathfrak{p p}^{(2)}$ and 


$$
\begin{array}{lcc}
d_{3} \equiv X^{2 \alpha-\alpha^{\prime}} Z^{\gamma+3 \gamma^{\prime}} \bmod (Y) & \text { and } & d_{3}^{\prime} \equiv Z^{3 \gamma+2 \gamma^{\prime}} \bmod (Y) \quad \text { if } 2 \beta<\beta^{\prime}, \\
d_{3} \equiv-Y^{4 \beta+2 \beta^{\prime}} Z^{2 \gamma^{\prime}-2 \gamma} \bmod (X) & \text { and } & d_{3}^{\prime} \equiv Y^{2 \beta-\beta^{\prime}} Z^{3 \gamma+2 \gamma^{\prime}} \bmod (X) \\
& \text { if } 2 \alpha>\alpha^{\prime}>\alpha \text { and } 2 \beta \geq \beta^{\prime}, \\
d_{3} \equiv-X^{4 \alpha+2 \alpha^{\prime}} \bmod (Y) & \text { and } & d_{3}^{\prime} \equiv-X^{2 \alpha+3 \alpha^{\prime}} Z^{2 \gamma-\gamma^{\prime}} \bmod (Y) \\
& & \text { if } 2 \beta>\beta^{\prime}>\beta, \\
d_{3} \equiv-X^{4 \alpha+2 \alpha^{\prime}} \bmod (Z) & \text { and } & d_{3}^{\prime} \equiv-X^{2 \alpha^{\prime}-\alpha} Y^{3 \beta+2 \beta^{\prime}} \bmod (Z) \\
& & \text { if } 2 \beta \geq \beta^{\prime} \text { and } 2 \gamma>\gamma^{\prime}>\gamma, \\
d_{3} \equiv-X^{2 \alpha+3 \alpha^{\prime}} \bmod (Z) & \text { and } & d_{3}^{\prime} \in \mathfrak{p p} \mathfrak{p}^{(2)} \quad \text { if } 2 \alpha=\alpha^{\prime} \text { and } 2 \beta=\beta^{\prime}, \\
d_{3} \in \mathfrak{p p}^{(2)} & \text { and } & d_{3}^{\prime} \equiv Z^{7 \gamma}-X^{5 \alpha} \bmod (Y) \\
& & \text { if } \alpha=\alpha^{\prime}, 2 \beta=\beta^{\prime} \text { and } 2 \gamma=\gamma^{\prime} .
\end{array}
$$

Remark (5.5). When $M$ is not of any case stated in (5.4). $M$ satisfies the condition (2) of (4.3) so that $\mathfrak{p}^{(3)}=\mathfrak{p p}^{(2)}$.

Proof of Theorem (5.4).

(1) First we assume $2 \alpha<\alpha^{\prime}$. By (5.1) we have

$$
\begin{aligned}
X^{\alpha}\left(b d_{2}-X^{\alpha^{\prime}-2 \alpha} Z^{2 \gamma^{\prime}-2 \gamma} c^{3}\right) & =Y^{\beta}\left(Z^{\gamma^{\prime}-\gamma} c d_{2}-Y^{\beta^{\prime}-2 \beta} b^{3}\right) & & \text { if } 2 \beta<\beta^{\prime}, \\
& =Y^{\beta^{\prime}-\beta}\left(Y^{2 \beta-\beta^{\prime}} Z^{\gamma^{\prime}-\gamma} c d_{2}-b^{3}\right) & & \text { if } 2 \beta \geq \beta^{\prime} .
\end{aligned}
$$

Hence there exists $d_{3} \in \mathfrak{p}^{(3)}$ such that

$$
\begin{aligned}
b d_{2}-X^{\alpha^{\prime}-2 \alpha} Z^{2 \gamma^{\prime}-2 \gamma} c^{3} & =Y^{\beta} d_{3} & & \text { if } 2 \beta<\beta^{\prime}, \\
& =Y^{\beta^{\prime}-\beta} d_{3} & & \text { if } 2 \beta \geq \beta^{\prime} .
\end{aligned}
$$

As $\alpha<\alpha^{\prime}$, by (2.1) $d_{2} \equiv-Z^{\gamma+2 \gamma^{\prime}} \bmod (X)$ and so

$$
\begin{aligned}
d_{3} & \equiv Z^{\gamma+3 \gamma^{\prime}} \bmod (X) & & \text { if } 2 \beta<\beta^{\prime}, \\
& \equiv Y^{2 \beta-\beta^{\prime}} Z^{\gamma+3 \gamma^{\prime}} \bmod (X) & & \text { if } 2 \beta \geq \beta^{\prime} .
\end{aligned}
$$

We put $I:=\left(d_{3}\right)+\mathfrak{p p}^{(2)}\left(\supseteq \mathfrak{p}^{3}\right)$. Then if $2 \beta<\beta^{\prime}$, we have

$$
\begin{aligned}
(X)+I=\left(X, Z^{\gamma+3 \gamma^{\prime}}, Y^{3 \beta} Z^{3 \gamma^{\prime}},\right. & Y^{\beta+\beta^{\prime}} Z^{\gamma+2 \gamma^{\prime}}, Y^{3 \beta+\beta^{\prime}} Z^{2 \gamma^{\prime}}, \\
& \left.Y^{2 \beta+2 \beta^{\prime}} Z^{\gamma+\gamma^{\prime}}, Y^{3 \beta+2 \beta^{\prime}} Z^{\gamma^{\prime}}, Y^{3 \beta+3 \beta^{\prime}}\right) .
\end{aligned}
$$

Hence $\ell_{A}(A /(X)+I)=6 \beta \gamma+6 \beta \gamma^{\prime}+6 \beta^{\prime} \gamma^{\prime}$. On the other hand we have by the additive formula $[18$, p. 126] of multiplicity that

$$
\begin{aligned}
\ell_{A}\left(A /(X)+\mathfrak{p}^{(3)}\right) & =e_{X A}\left(A / \mathfrak{p}^{(3)}\right) \\
& =\ell_{A_{\mathfrak{p}}}\left(A_{\mathfrak{p}} / \mathfrak{p}^{3} A_{\mathfrak{p}}\right) \cdot e_{X A}(A / \mathfrak{p}) \\
& =6 \cdot \ell_{A}(A /(X)+\mathfrak{p}) \\
& =6 \beta \gamma+6 \beta \gamma^{\prime}+6 \beta^{\prime} \gamma^{\prime} .
\end{aligned}
$$


Thus we get $\ell_{A}(A /(X)+I)=\ell_{A}\left(A /(X)+\mathfrak{p}^{(3)}\right)$ and consequently $(X)+I=$ $(X)+\mathfrak{p}^{(3)}$. Hence $\mathfrak{p}^{(3)}=I+(X) \cap \mathfrak{p}^{(3)}$, so we have $\mathfrak{p}^{(3)}=I$ by Nakayama's lemma. Similarly we get $\mathfrak{p}^{(3)}=I$ for the case $2 \beta \geq \beta^{\prime}$, too.

Secondly, assume $2 \gamma<\gamma^{\prime}$. Then by (5.3) we have

$$
\begin{aligned}
X^{\alpha}\left(b d_{2}+X^{\alpha^{\prime}-\alpha} Z^{\gamma^{\prime}-2 \gamma} a c^{2}\right) & =Y^{\beta}\left(-Z^{\gamma^{\prime}-2 \gamma} a^{2} c-Y^{\beta^{\prime}-2 \beta} b^{3}\right) & & \text { if } 2 \beta<\beta^{\prime}, \\
& =Y^{\beta^{\prime}-\beta}\left(-Y^{2 \beta-\beta^{\prime}} Z^{\gamma^{\prime}-2 \gamma} a^{2} c-b^{3}\right) & & \text { if } 2 \beta \geq \beta^{\prime} .
\end{aligned}
$$

Hence there exists $d_{3} \in \mathfrak{p}^{(3)}$ satisfying

$$
\begin{aligned}
b d_{2}+X^{\alpha^{\prime}-\alpha} Z^{\gamma^{\prime}-2 \gamma} a c^{2} & =Y^{\beta} d_{3} & & \text { if } 2 \beta<\beta^{\prime}, \\
& =Y^{\beta^{\prime}-\beta} d_{3} & & \text { if } 2 \beta \geq \beta^{\prime}
\end{aligned}
$$

so that

$$
\begin{aligned}
d_{3} & \equiv-X^{2 \alpha+3 \alpha^{\prime}} Y^{\beta^{\prime}-2 \beta} \bmod (Z) & & \text { if } 2 \beta<\beta^{\prime} \\
& \equiv-X^{2 \alpha+3 \alpha^{\prime}} \bmod (Z) & & \text { if } 2 \beta \geq \beta^{\prime}
\end{aligned}
$$

Let $I=\left(d_{3}\right)+\mathfrak{p p}^{(2)}$. Then similarly as in the proof for the case where $2 \alpha<\alpha^{\prime}$, we get

$$
\begin{aligned}
\ell_{A}(A /(Z)+I) & =6 \alpha^{\prime} \beta+6 \alpha^{\prime} \beta^{\prime}+6 \alpha \beta^{\prime} \\
& =\ell_{A}\left(A /(Z)+\mathfrak{p}^{(3)}\right),
\end{aligned}
$$

whence $I=\mathfrak{p}^{(3)}$ by Nakayama's lemma.

(2) Since $2 \alpha \geq \alpha^{\prime}$, we have by (5.1) that

$$
\begin{aligned}
X^{\alpha^{\prime}-\alpha}\left(X^{2 \alpha-\alpha^{\prime}} b d_{2}-Z^{2 \gamma^{\prime}-2 \gamma} c^{3}\right) & =Y^{\beta}\left(Z^{\gamma^{\prime}-\gamma} c d_{2}-Y^{\beta^{\prime}-2 \beta} b^{3}\right) & & \text { if } 2 \beta<\beta^{\prime}, \\
& =Y^{\beta^{\prime}-\beta}\left(Y^{2 \beta-\beta^{\prime}} Z^{\gamma^{\prime}-\gamma} c d_{2}-b^{3}\right) & & \text { if } 2 \beta \geq \beta^{\prime} .
\end{aligned}
$$

On the other hand since $2 \gamma \geq \gamma^{\prime}$, we get by (5.2) that

$$
\begin{aligned}
X^{\alpha}\left(Z^{2 \gamma-\gamma^{\prime}} b d_{2}+X^{\alpha^{\prime}-\alpha} a c^{2}\right) & =Y^{\beta}\left(-a^{2} c-Y^{\beta^{\prime}-2 \beta} Z^{2 \gamma-\gamma^{\prime}} b^{3}\right) & & \text { if } 2 \beta<\beta^{\prime} . \\
& =Y^{\beta^{\prime}-\beta}\left(-Y^{2 \beta-\beta^{\prime}} a^{2} c-Z^{2 \gamma-\gamma^{\prime}} b^{3}\right) & & \text { if } 2 \beta \geq \beta^{\prime} .
\end{aligned}
$$

Hence there exist $d_{3} \in \mathfrak{p}^{(3)}$ and $d_{3}^{\prime} \in \mathfrak{p}^{(3)}$ such that

$$
\begin{aligned}
X^{\alpha^{\prime}-\alpha} d_{3}=Z^{\gamma^{\prime}-\gamma} c d_{2}-Y^{\beta^{\prime}-2 \beta} b^{3} & \text { if } 2 \beta<\beta^{\prime}, \\
=Y^{2 \beta-\beta^{\prime}} Z^{\gamma^{\prime}-\gamma} c d_{2}-b^{3} & \text { if } 2 \beta \geq \beta^{\prime}, \\
X^{2 \alpha-\alpha^{\prime}} b d_{2}-Z^{2 \gamma^{\prime}-2 \gamma} c^{3}=Y^{\beta} d_{3} & \text { if } 2 \beta<\beta^{\prime}, \\
=Y^{\beta^{\prime}-\beta} d_{3} & \text { if } 2 \beta \geq \beta^{\prime},
\end{aligned}
$$

and 


$$
\begin{aligned}
X^{\alpha} d_{3}^{\prime}=-a^{2} c-Y^{\beta^{\prime}-2 \beta} Z^{2 \gamma-\gamma^{\prime}} b^{3} & \text { if } 2 \beta<\beta^{\prime}, \\
=-Y^{2 \beta-\beta^{\prime}} a^{2} c-Z^{2 \gamma-\gamma^{\prime}} b^{3} & \text { if } 2 \beta \geq \beta^{\prime}, \\
Z^{2 \gamma-\gamma^{\prime}} b d_{2}+X^{\alpha^{\prime}-\alpha} a c^{2}=Y^{\beta} d_{3}^{\prime} & \text { if } 2 \beta<\beta^{\prime}, \\
=Y^{\beta^{\prime}-\beta} d_{3}^{\prime} & \text { if } 2 \beta \geq \beta^{\prime} .
\end{aligned}
$$

Then putting $I=\left(d_{3}, d_{3}^{\prime}\right)+\mathfrak{p p}^{(2)}$, we will get, similarly as in the proof of the assertion (1), that $I=\mathfrak{p}^{(3)}$ for each case in the statement (2). The detail shall be left to the readers.

Corollary (5.6). Suppose that $M$ has type (I). Then

$$
\begin{aligned}
\mu_{A}\left(\mathfrak{p}^{(3)} / \mathfrak{p p ^ { ( 2 ) }}\right) \leq 1 & \text { if } 2 \alpha \leq \alpha^{\prime} \text { or } 2 \gamma \leq \gamma^{\prime}, \\
\leq 2 & \text { if } 2 \alpha>\alpha^{\prime} \text { and } 2 \gamma>\gamma^{\prime} .
\end{aligned}
$$

Let us now assume that $M$ has type (II). Then since $Y^{\beta} a+Z^{\gamma} b+$ $X^{\alpha^{\prime}} c=0$, we get by the equations given in (2.3) that

$$
\begin{aligned}
& c\left(Z^{\gamma^{\prime}-\gamma} a c-Y^{\beta^{\prime}-\beta} b^{2}\right)=X^{\alpha^{\prime}} c d_{2}=d_{2}\left(-Y^{\beta} a-Z^{\gamma} b\right), \\
& a\left(X^{\alpha-\alpha^{\prime}} a b-Z^{\gamma^{\prime}-\gamma} c^{2}\right)=Y^{\beta} a d_{2}=d_{2}\left(-Z^{\gamma} b-X^{\alpha^{\prime}} c\right) \text { and } \\
& b\left(Y^{\beta^{\prime}-\beta} b c-X^{\alpha-\alpha^{\prime}} a^{2}\right)=Z^{\gamma} b d_{2}=d_{2}\left(-Y^{\beta} a-X^{\alpha^{\prime}} c\right) .
\end{aligned}
$$

Hence we have

$$
\begin{aligned}
& Y^{\beta^{\prime}-\beta} b^{2} c-Y^{\beta} a d_{2}=Z^{\gamma^{\prime}-\gamma} a c^{2}+Z^{\gamma} b d_{2}, \\
& Z^{\gamma^{\prime}-\gamma} a c^{2}-Z^{\gamma} b d_{2}=X^{\alpha-\alpha^{\prime}} a^{2} b+X^{\alpha^{\prime}} c d_{2} \text { and } \\
& X^{\alpha-\alpha^{\prime}} a^{2} b-X^{\alpha^{\prime}} c d_{2}=Y^{\beta^{\prime}-\beta} b^{2} c+Y^{\beta} a d_{2} .
\end{aligned}
$$

We furthermore assume that

$$
\alpha \neq 2 \alpha^{\prime}, \quad 2 \beta \neq \beta^{\prime} \quad \text { or } \quad 2 \gamma \neq \gamma^{\prime}
$$

(cf. (2.1), (3)). Then after suitable permutations of columns, $M$ is assumed to satisfy one of the followings:

(i) $2 \alpha^{\prime}<\alpha, 2 \beta<\beta^{\prime}$ and $2 \gamma<\gamma^{\prime}$;

(ii) $2 \alpha^{\prime}<\alpha, 2 \beta<\beta^{\prime}$ and $2 \gamma \geq \gamma^{\prime}$;

(iii) $2 \alpha^{\prime}<\alpha, 2 \beta \geq \beta^{\prime}$ and $2 \gamma \geq \gamma^{\prime}$;

(iv) $2 \alpha^{\prime}>\alpha, 2 \beta \geq \beta^{\prime}$ and $2 \gamma \geq \gamma^{\prime}$.

THeOREM (5.10). Suppose that $M$ has type (II) and satisfies one of the above conditions (i), (ii), (iii) and (iv). Then we have the following assertions.

(1) If $\frac{1}{2} \in A$, then there exist $d_{3}, d_{3}^{\prime}$ and $d_{3}^{\prime \prime}$ in $\mathfrak{p}^{(3)}$ such that 


$$
\begin{aligned}
d_{3} \equiv & 2 Y^{\beta+2 \beta^{\prime}} Z^{2 \gamma^{\prime}-\gamma} \bmod (X), \quad d_{3} \equiv-Y^{2 \beta+3 \beta^{\prime}} Z^{\gamma^{\prime}-2 \gamma} \bmod (X) \text { and } \\
& d_{3}^{\prime \prime} \equiv Y^{\beta+3 \beta^{\prime}} Z^{\gamma^{\prime}-\gamma} \bmod (X), \quad \text { if } M \text { is of case (i); } \\
d_{3} \equiv & 2 Y^{\beta+2 \beta^{\prime}} Z^{\gamma+\gamma^{\prime}} \bmod (X), \quad d_{3}^{\prime} \equiv-Y^{2 \beta+3 \beta^{\prime}} \bmod (X) \text { and } \\
& d_{3}^{\prime \prime} \equiv Y^{\beta+3 \beta^{\prime}} Z^{\gamma^{\prime}-\gamma} \bmod (X), \quad \text { if } M \text { is of case (ii); } \\
d_{3} \equiv & 2 Y^{3 \beta+\beta^{\prime}} Z^{\gamma+\gamma^{\prime}} \bmod (X), \quad d_{3}^{\prime} \equiv-Y^{2 \beta+3 \beta^{\prime}} \bmod (X) \text { and } \\
& d_{3}^{\prime \prime} \equiv Y^{3 \beta+2 \beta^{\prime}} Z^{\gamma^{\prime}-\gamma} \bmod (X), \quad \text { if } M \text { is of case (iii); } \\
d_{3} \equiv & 2 Y^{3 \beta+\beta^{\prime}} Z^{\gamma+\gamma^{\prime}} \bmod (X), \quad d_{3}^{\prime} \equiv-Y^{\beta} Z^{3 \gamma+2 \gamma^{\prime}} \bmod (X) \text { and } \\
& d_{3}^{\prime \prime} \equiv-Y^{2 \beta-\beta^{\prime}} Z^{2 \gamma+3 \gamma^{\prime}} \bmod (X), \quad \text { if } M \text { is of case (iv). }
\end{aligned}
$$

We furthermore have $\mathfrak{p}^{(3)}=\left(d_{3}, d_{3}^{\prime}, d_{3}^{\prime \prime}\right)+\mathfrak{p p}^{(2)}$.

(2) If $\operatorname{ch} A=2$, then there exist $e_{3} \in \mathfrak{p}^{(3)}$ such that $\mathfrak{p}^{(3)}=\left(e_{3}\right)+\mathfrak{p p}^{(2)}$ and

$$
\begin{array}{ll}
e_{3} \equiv Y^{\beta+3 \beta^{\prime}} Z^{\gamma^{\prime}-2 \gamma} \bmod (X) & \text { if } M \text { is of case (i); } \\
e_{3} \equiv Y^{\beta+3 \beta^{\prime}} \bmod (X) & \text { if } M \text { is of case (ii); } \\
e_{3} \equiv-Y^{3 \beta+2 \beta^{\prime}} \bmod (X) & \text { if } M \text { is of case (iii); } \\
e_{3} \equiv-Y^{2 \beta-\beta^{\prime}} Z^{3 \gamma+2 \gamma^{\prime}} \bmod (X) & \text { if } M \text { is of case (iv). }
\end{array}
$$

Proof. (1) Let us assume the case (i). Then by (5.7), (5.8) and (5.9)

we have

$$
\begin{aligned}
& Y^{\beta}\left(Y^{\beta^{\prime}-2 \beta} b^{2} c-a d_{2}\right)=Z^{\gamma}\left(z^{\gamma^{\prime}-2 \gamma} a c^{2}+b d_{2}\right), \\
& Z^{\gamma}\left(Z^{\gamma^{\prime}-2 \gamma} a c^{2}-b d_{2}\right)=X^{\alpha^{\prime}}\left(X^{\alpha-2 \alpha^{\prime}} a^{2} b+c d_{2}\right) \text { and } \\
& X^{\alpha^{\prime}}\left(X^{\alpha-2 \alpha^{\prime}} a^{2} b-c d_{2}\right)=Y^{\beta}\left(Y^{\beta^{\prime}-2 \beta} b^{2} c+a d_{2}\right) .
\end{aligned}
$$

Hence there exist $d_{3}, d_{3}^{\prime}$ and $d_{3}^{\prime \prime}$ in $\mathfrak{p}^{(3)}$ such that

$$
\begin{aligned}
& Y^{\beta} d_{3}=Z^{\gamma^{\prime}-2 \gamma} a c^{2}+b d_{2}, \\
& Z^{\gamma} d_{3}^{\prime}=X^{\alpha-2 \alpha^{\prime}} a^{2} b+c d_{2} \text { and } \\
& Y^{\beta} d_{3}^{\prime \prime}=X^{\alpha-2 \alpha^{\prime}} a^{2} b-c d_{2} .
\end{aligned}
$$

Thus

$$
\begin{aligned}
& d_{3} \equiv 2 Y^{\beta+2 \beta^{\prime}} Z^{2 \gamma^{\prime}-\gamma} \bmod (X), \\
& d_{3}^{\prime} \equiv-Y^{2 \beta+3 \beta^{\prime}} Z^{\gamma^{\prime}-2 \gamma} \bmod (X) \text { and } \\
& d_{3}^{\prime \prime} \equiv Y^{\beta+3 \beta^{\prime}} Z^{\gamma^{\prime}-\gamma} \bmod (X) .
\end{aligned}
$$

Let $I=\left(d_{3}, d_{3}^{\prime}, d_{3}^{\prime \prime}\right)+\mathfrak{p p}^{(2)}$. Then we have

$$
\begin{aligned}
(X)+I= & (X)+\left(Z^{3 \gamma+3 \gamma^{\prime}}, Y^{\beta} Z^{2 \gamma+3 \gamma^{\prime}}, Y^{2 \beta} Z^{\gamma+3 \gamma^{\prime}}, Y^{3 \beta} Z^{3 \gamma^{\prime}},\right. \\
& Y^{\beta+\beta^{\prime}} Z^{2 \gamma+2 \gamma^{\prime}}, Y^{2 \beta+\beta^{\prime}} Z^{\gamma+2 \gamma^{\prime}}, Y^{3 \beta+\beta^{\prime}} Z^{2 \gamma^{\prime}}, Y^{\beta+2 \beta^{\prime}} Z^{2 \gamma^{\prime}-\gamma}, \\
& Y^{2 \beta+2 \beta^{\prime}} Z^{\gamma+\gamma^{\prime}}, Y^{3 \beta+2 \beta^{\prime}} Z^{\gamma^{\prime}}, Y^{\beta+3 \beta^{\prime}} Z^{\gamma^{\prime}-\gamma}, Y^{2 \beta+3 \beta^{\prime}} Z^{\gamma^{\prime}-2 \gamma}, \\
& \left.Y^{3 \beta+3 \beta^{\prime}}\right) .
\end{aligned}
$$


Hence $\ell_{A}(A /(X)+I)=6\left(\beta \gamma+\beta \gamma^{\prime}+\beta^{\prime} \gamma^{\prime}\right)=\ell_{A}\left(A /(X)+\mathfrak{p}^{(3)}\right)$, which implies $(X)+I=(X)+\mathfrak{p}^{(3)}$ so that $\mathfrak{p}^{(3)}=I$ by Nakayama's lemma. The same proof works for the other case, too. For instance, in the case (ii), we get by (5.7), (5.8) and (5.9) that

$$
\begin{aligned}
& Y^{\beta}\left(Y^{\beta^{\prime}-2 \beta} b^{2} c-a d_{2}\right)=Z^{\gamma^{\prime}-\gamma}\left(a c^{2}+Z^{2 \gamma-\gamma^{\prime}} b d_{2}\right), \\
& Z^{\gamma^{\prime}-\gamma}\left(a c^{2}-Z^{2 \gamma-\gamma^{\prime}} b d_{2}\right)=X^{\alpha^{\prime}}\left(X^{\alpha-2 \alpha^{\prime}} a^{2} b+c d_{2}\right) \text { and } \\
& X^{\alpha^{\prime}}\left(X^{\alpha-2 \alpha^{\prime}} a^{2} b-c d_{2}\right)=Y^{\beta}\left(Y^{\beta^{\prime}-2 \beta} b^{2} c+a d_{2}\right) .
\end{aligned}
$$

Hence there exist $d_{3}, d_{3}^{\prime}$ and $d_{3}^{\prime \prime}$ in $\mathfrak{p}^{(3)}$ satisfying

$$
\begin{aligned}
& Y^{\beta} d_{3}=a c^{2}+Z^{2 \gamma-\gamma^{\prime}} b d_{2}, \\
& Z^{\gamma^{\prime}-\gamma} d_{3}^{\prime}=X^{\alpha-2 \alpha^{\prime}} a^{2} b+c d_{2} \text { and } \\
& Y^{\beta} d_{3}^{\prime \prime}=X^{\alpha-2 \alpha^{\prime}} a^{2} b-c d_{2}
\end{aligned}
$$

so that

$$
\begin{aligned}
& d_{3} \equiv 2 Y^{\beta+2 \beta^{\prime}} Z^{\gamma+\gamma^{\prime}} \bmod (X) \\
& d_{3}^{\prime} \equiv-Y^{2 \beta+3 \beta^{\prime}} \bmod (X) \text { and } \\
& d_{3}^{\prime \prime} \equiv Y^{\beta+3 \beta^{\prime}} Z^{\gamma^{\prime}-\gamma} \bmod (X) .
\end{aligned}
$$

And therefore we can prove $\mathfrak{p}^{(3)}=\left(d_{3}, d_{3}^{\prime}, d_{3}^{\prime \prime}\right)+\mathfrak{p p}^{(2)}$ by showing that $\ell_{A}\left(A /(X)+\mathfrak{p}^{(3)}\right)=\ell_{A}\left(A /\left(X, d_{3}, d_{3}^{\prime}, d_{3}^{\prime \prime}\right)+\mathfrak{p p}^{(2)}\right)$ similarly as above. We would like to leave to the readers the rest of Proof of Theorem (5.10), (1).

(2) Notice that

$$
\begin{aligned}
& X^{\alpha} a+Y^{\beta^{\prime}} b+Z^{\gamma^{\prime}} c=0 \text { and } \\
& Y^{\beta} a+Z^{\gamma} b+X^{\alpha^{\prime}} c=0 .
\end{aligned}
$$

Then since $\operatorname{ch} A=2$, by (5.12) we get

$$
Y^{2 \beta} a^{2}+Z^{2 \gamma} b^{2}+X^{2 \alpha^{\prime}} c^{2}=0 .
$$

Suppose the case (i). Then by (5.11) and (5.13) we have

$$
\begin{aligned}
\left(Y^{2 \beta} a^{2}+X^{2 \alpha^{\prime}} c^{2}\right) Z^{\gamma^{\prime}-2 \gamma} c & =-Z^{\gamma^{\prime}} b^{2} c \\
& =\left(X^{\alpha} a+Y^{\beta^{\prime}} b\right) b^{2},
\end{aligned}
$$

whence

$$
X^{2 \alpha^{\prime}} Z^{\gamma^{\prime}-2 \gamma} c^{3}-X^{\alpha} a b^{2}=Y^{\beta^{\prime}} b^{3}-Y^{2 \beta} Z^{\gamma^{\prime}-2 \gamma} a^{2} c .
$$

Since $2 \alpha^{\prime}<\alpha$ and $2 \beta<\beta^{\prime}$ by our assumption, we get

$$
X^{2 \alpha^{\prime}}\left(Z^{\gamma^{\prime}-2 \gamma} c^{3}-X^{\alpha-2 \alpha^{\prime}} a b^{2}\right)=Y^{2 \beta}\left(Y^{\beta^{\prime}-2 \beta} b^{3}-Z^{\gamma^{\prime}-2 \gamma} a^{2} c\right)
$$


from which we find $e_{3} \in \mathfrak{p}^{(3)}$ satisfying

$$
Y^{2 \beta} e_{3}=Z^{\gamma^{\prime}-2 \gamma} c^{3}-X^{\alpha-2 \alpha^{\prime}} a b^{2} .
$$

Notice that $e_{3} \equiv Y^{\beta+3 \beta^{\prime}} Z^{\gamma^{\prime}-2 \gamma} \bmod (X)$. Then because

$$
\begin{aligned}
\ell_{A}\left(A /\left(X, e_{3}\right)+\mathfrak{p p}^{(2)}\right) & =6\left(\beta \gamma+\beta \gamma^{\prime}+\beta^{\prime} \gamma^{\prime}\right) \\
& =\ell_{A}\left(A /(X)+\mathfrak{p}^{(3)}\right),
\end{aligned}
$$

we see $\left(X, e_{3}\right)+\mathfrak{p p}^{(2)}=(X)+\mathfrak{p}^{(3)}$ so that $\left(e_{3}\right)+\mathfrak{p p}^{(2)}=\mathfrak{p}^{(3)}$. A similar proof works for the other cases (ii), (iii) and (iv), whose detail shall be left to the readers.

\section{$\S 6$. The generation of $R_{s}(\mathfrak{p})$ in degree three}

The purpose of this section is to characterize the prime ideals $\mathfrak{p}$ whose symbolic Rees algebras $R_{s}(\mathfrak{p})$ are generated in degree three. Let us maintain the same assumption as in Section 5 .

First we shall discuss the case where $M$ has type (I) and the results of this case are summarized into the next

Theorem (6.1). Suppose that $M$ has type (I). Then the following assertions are equivalent.

(1) $R_{s}(\mathfrak{p})=A\left[\mathfrak{p} t, \mathfrak{p}^{(2)} t^{2}, \mathfrak{p}^{(3)} t^{3}\right]$.

(2) $M$ satisfies one of the following conditions.

(i) $2 \alpha \leq \alpha^{\prime}$ and $2 \beta=\beta^{\prime}$.

(ii) $2 \beta=\beta^{\prime}$ and $2 \gamma \leq \gamma^{\prime}$.

(iii) $2 \alpha=\alpha^{\prime}$ and $\gamma=\gamma^{\prime}$.

(iv) $2 \alpha>\alpha^{\prime}, 2 \beta \geq \beta^{\prime}$ and $\gamma=\gamma^{\prime}$.

(v) $\beta=\beta^{\prime}$.

(vi) $\alpha=\alpha^{\prime}$ and $\gamma=\gamma^{\prime}$.

When this is the case, $R_{s}(\mathfrak{p})$ is a Gorenstein ring.

We divide the proof of (6.1) according to the cases stated in (5.4). The proof will be finished by the end of Proposition (6.11).

Proposition (6.2). Suppose that $M$ is of type (I) and satisfies $2 \alpha<\alpha^{\prime}$ and $2 \beta<\beta^{\prime}$. Then $R_{s}(\mathfrak{p}) \neq A\left[\mathfrak{p} t, \mathfrak{p}^{(2)} t^{2}, \mathfrak{p}^{(3)} t^{3}\right]$.

Proof. It is enough to show that $\mathfrak{p}^{(4)} \neq\left[\mathfrak{p}^{(2)}\right]^{2}+\mathfrak{p} \mathfrak{p}^{(3)}$. Assume the contrary and we have $\ell_{A}\left(A /(X)+\mathfrak{p}^{(4)}\right)=\ell_{A}\left(A /(X)+\left[\mathfrak{p}^{(2)}\right]^{2}+\mathfrak{p p}^{(3)}\right)$. On the other hand by (2.2) and (5.4) we get 


$$
\begin{aligned}
(X)+\left[\mathfrak{p}^{(2)}\right]^{2}+\mathfrak{p p}^{(3)}= & (X)+\left(Z^{2 \gamma+4 \gamma^{\prime}}, Y^{\beta} Z^{\gamma+4 \gamma^{\prime}}, Y^{4 \beta} Z^{4 \gamma^{\prime}}, Y^{\beta+\beta^{\prime}} Z^{\gamma+3 \gamma^{\prime}},\right. \\
& Y^{4 \beta+\beta^{\prime}} Z^{3 \gamma^{\prime}}, Y^{2 \beta+2 \beta^{\prime}} Z^{\gamma+2 \gamma^{\prime}}, Y^{4 \beta+2 \beta^{\prime}} Z^{2 \gamma^{\prime}}, Y^{3 \beta+3 \beta^{\prime}} Z^{\gamma+\gamma^{\prime}}, \\
& \left.Y^{4 \beta+3 \beta^{\prime}} Z^{\gamma^{\prime}}, Y^{4 \beta+4 \beta^{\prime}}\right) .
\end{aligned}
$$

Hence if $3 \beta>\beta^{\prime}$, we have

$$
\begin{aligned}
(X)+\left[\mathfrak{p}^{(2)}\right]^{2}+\mathfrak{p p}^{(3)}= & (X)+\left(Z^{2 \gamma+4 \gamma^{\prime}}, Y^{\beta} Z^{\gamma+4 \gamma^{\prime}}, Y^{\beta+\beta^{\prime}} Z^{\gamma+3 \gamma^{\prime}}, Y^{4 \beta+\beta^{\prime}} Z^{3 \gamma^{\prime}}\right. \\
& Y^{2 \beta+2 \beta^{\prime}} Z^{\gamma+2 \gamma^{\prime}}, Y^{4 \beta+2 \beta^{\prime}} Z^{2 \gamma^{\prime}}, Y^{3 \beta+3 \beta^{\prime}} Z^{\gamma+\gamma^{\prime}}, Y^{4 \beta+3 \beta^{\prime}} Z^{\gamma^{\prime}} \\
& \left.Y^{4 \beta+4 \beta^{\prime}}\right) .
\end{aligned}
$$

because $Y^{4 \beta} Z^{4 \gamma^{\prime}} \in\left(Y^{\beta+\beta^{\prime}} Z^{\gamma+3 r^{\prime}}\right)$. Therefore, counting the number of monomials of $Y$ and $Z$ that are not in $(X)+\left[\mathfrak{p}^{(2)}\right]^{2}+\mathfrak{p p}^{(3)}$, we find

$$
\begin{aligned}
\ell_{A}\left(A /(X)+\left[\mathfrak{p}^{(2)}\right]^{2}+\mathfrak{p p ^ { ( 3 ) }}\right) & =11 \beta \gamma+10 \beta \gamma^{\prime}+10 \beta^{\prime} \gamma^{\prime} & & \text { if } 3 \beta \leq \beta^{\prime}, \\
& =8 \beta \gamma+10 \beta \gamma^{\prime}+10 \beta^{\prime} \gamma^{\prime}+\beta^{\prime} \gamma & & \text { if } 3 \beta>\beta^{\prime},
\end{aligned}
$$

while

$$
\begin{aligned}
\ell_{A}\left(A /(X)+\mathfrak{p}^{(4)}\right) & =\ell_{A_{\mathfrak{p}}}\left(A_{\mathfrak{p}} / \mathfrak{p}^{4} A_{\mathfrak{p}}\right) \cdot \ell_{A}(A /(X)+\mathfrak{p}) \\
& =10\left(\beta \gamma+\beta \gamma^{\prime}+\beta^{\prime} \gamma^{\prime}\right)
\end{aligned}
$$

by the additive formula [18, p. 126] of multiplicity. Thus we have

$$
\begin{aligned}
10\left(\beta \gamma+\beta \gamma^{\prime}+\beta^{\prime} \gamma^{\prime}\right) & =11 \beta \gamma+10 \beta \gamma^{\prime}+10 \beta^{\prime} \gamma^{\prime} & & \text { if } 3 \beta \leq \beta^{\prime}, \\
& =8 \beta \gamma+10 \beta \gamma^{\prime}+10 \beta^{\prime} \gamma^{\prime}+\beta^{\prime} \gamma & & \text { if } 3 \beta>\beta^{\prime},
\end{aligned}
$$

from which we conclude that $\beta \gamma=0$ or $2 \beta=\beta^{\prime}$. This contradicts our assumption that $2 \beta<\beta^{\prime}$. Hence $\mathfrak{p}^{(4)} \neq\left[\mathfrak{p}^{(2)}\right]^{2}+\mathfrak{p p}^{(3)}$.

Proposition (6.3). Suppose that $M$ is of type (I) and satisfies $2 \alpha<\alpha^{\prime}$ and $2 \beta \geq \beta^{\prime}$. Then $\mathfrak{p}^{(4)}=\left[\mathfrak{p}^{(2)}\right]^{2}+\mathfrak{p p}^{(3)}$ and the following conditions are equivalent.

(1) $R_{s}(\mathfrak{p})=A\left[\mathfrak{p} t, \mathfrak{p}^{(2)} t^{2}, \mathfrak{p}^{(3)} t^{3}\right]$.

(2) $\mathfrak{p}^{(5)}=\mathfrak{p}^{(2)} \mathfrak{p}^{(3)}$.

(3) $2 \beta=\beta^{\prime}$ or $\beta=\beta^{\prime}$.

When this is the case, $R_{s}(\mathfrak{p})$ is a Gorenstein ring.

Proof. By (2.2) and (5.4) we have

$$
\begin{aligned}
(X)+\left[\mathfrak{p}^{(2)}\right]^{2}+\mathfrak{p p} \mathfrak{p}^{(3)}= & (X)+\left(Z^{2 \gamma+4 \gamma^{\prime}}, Y^{3 \beta-\beta^{\prime}} Z^{\gamma+4 \gamma^{\prime}}, Y^{\beta+\beta^{\prime}} Z^{2 \gamma+3 \gamma^{\prime}}, Y^{3 \beta} Z^{\gamma+3 \gamma^{\prime}},\right. \\
& Y^{2 \beta+2 \beta^{\prime}} Z^{\gamma+2 \gamma^{\prime}}, Y^{4 \beta+2 \beta^{\prime}} Z^{2 \gamma^{\prime}}, Y^{3 \beta+3 \beta^{\prime}} Z^{\gamma+\gamma^{\prime}}, Y^{4 \beta+3 \beta^{\prime}} Z^{\gamma^{\prime}}, \\
& \left.Y^{4 \beta+4 \beta^{\prime}}\right) .
\end{aligned}
$$

whence 


$$
\ell_{A}\left(A /(X)+\left[\mathfrak{p}^{(2)}\right]^{2}+\mathfrak{p p}{ }^{(3)}\right)=10 \beta \gamma+10 \beta \gamma^{\prime}+10 \beta^{\prime} \gamma^{\prime}
$$

Because $\ell_{A}\left(A /(X)+\mathfrak{p}^{(4)}\right)=10 \beta \gamma+10 \beta \gamma^{\prime}+10 \beta^{\prime} \gamma^{\prime}$ (cf. Proof of (6.2)), we get $(X)+\left[\mathfrak{p}^{(2)}\right]^{2}+\mathfrak{p} \mathfrak{p}^{(3)}=(X)+\mathfrak{p}^{(4)}$ so that $\left[\mathfrak{p}^{(2)}\right]^{2}+\mathfrak{p p}^{(3)}=\mathfrak{p}^{(4)}$ as required.

(1) $\Rightarrow(2)$ This is obvious.

$(2) \Rightarrow(3)$ Since by (2.2) and (5.4) we explicitly have a system of generators for the ideal $(X)+\mathfrak{p}^{(2)} \mathfrak{p}^{(3)}$, we get

$$
\begin{aligned}
\ell_{A}\left(A /(X)+\mathfrak{p}^{(2)} \mathfrak{p}^{(3)}\right) & =17 \beta \gamma+15 \beta \gamma^{\prime}+15 \beta^{\prime} \gamma^{\prime}-\beta^{\prime} \gamma & & \text { if } 3 \beta<2 \beta^{\prime}, \\
& =14 \beta \gamma+15 \beta \gamma^{\prime}+15 \beta^{\prime} \gamma^{\prime}+\beta^{\prime} \gamma & & \text { if } 3 \beta \geq 2 \beta^{\prime} .
\end{aligned}
$$

As $\ell_{A}\left(A /(X)+\mathfrak{p}^{(5)}\right)=\ell_{A}\left(A /(X)+\mathfrak{p}^{(2)} \mathfrak{p}^{(3)}\right)$ by our assumption and as

$$
\begin{aligned}
\ell_{A}\left(A /(X)+\mathfrak{p}^{(5)}\right) & =\ell_{A_{\mathfrak{p}}}\left(A_{\mathfrak{p}} / \mathfrak{p}^{5} A_{\mathfrak{p}}\right) \cdot \ell_{A}(A /(X)+\mathfrak{p}) \\
& =15\left(\beta \gamma+\beta \gamma^{\prime}+\beta^{\prime} \gamma^{\prime}\right),
\end{aligned}
$$

we have

$$
\begin{aligned}
15\left(\beta \gamma+\beta \gamma^{\prime}+\beta^{\prime} \gamma^{\prime}\right) & =17 \beta \gamma+15 \beta \gamma^{\prime}+15 \beta^{\prime} \gamma^{\prime}-\beta^{\prime} \gamma & & \text { if } 3 \beta<2 \beta^{\prime}, \\
& =14 \beta \gamma+15 \beta \gamma^{\prime}+15 \beta^{\prime} \gamma^{\prime}+\beta^{\prime} \gamma & & \text { if } 3 \beta \geq 2 \beta^{\prime} .
\end{aligned}
$$

Hence $2 \beta=\beta^{\prime}$ or $\beta=\beta^{\prime}$ by these equations.

$(3) \Rightarrow(1)$ By (4.3) we have only to consider the case where $2 \beta=\beta^{\prime}$. Let $d_{3}$ be the element in (1) of (5.4). Then by [11, Theorem 3.1] $R_{s}(\mathfrak{p})$ is a Noetherian ring, because

$$
\begin{aligned}
\ell_{A}\left(A /\left(X, c, d_{3}\right)\right) & =\ell_{A}\left(A /\left(X, Y^{3 \beta}, Z^{\gamma+3 r^{\prime}}\right)\right) \\
& =3\left(\beta \gamma+3 \beta \gamma^{\prime}\right) \\
& =1 \cdot 3 \cdot \ell_{A}(A /(X)+\mathfrak{p}) .
\end{aligned}
$$

We want to check that $R_{s}(\mathfrak{p})$ is a Gorenstein ring. Let $I=(c)+\mathfrak{p}^{(2)}$. Then since

$$
(X)+I=(X)+\left(Z^{\gamma+2 \gamma^{\prime}}, Y^{2 \beta} Z^{2 \gamma^{\prime}}, Y^{3 \beta}\right),
$$

we have $\ell_{A}(A /(X)+I)=2 \beta \gamma+6 \beta \gamma^{\prime}$, while

$$
\begin{aligned}
e_{X A}(A / I) & =\ell_{A_{\mathfrak{p}}}\left(A_{\mathfrak{p}} / c A_{\mathfrak{p}}+\mathfrak{p}^{2} A_{\mathfrak{p}}\right) \cdot \ell_{A}(A /(X)+\mathfrak{p}) \\
& =2\left(\beta \gamma+3 \beta \gamma^{\prime}\right) .
\end{aligned}
$$

Consequently we get $\ell_{A}(A /(X)+I)=e_{X A}(A / I)$, whence the ring $A / I$ is Cohen-Macaulay. Thus $R_{s}(\mathfrak{p})$ is a Gorenstein ring (cf. [5, Theorem 1.1]), which completes the proof of (6.3). 
Proposition (6.4). Suppose that $M$ is of type (I) and satisfies $2 \beta<\beta^{\prime}$ and $2 \gamma<\gamma^{\prime}$. Then $R_{s}(\mathfrak{p}) \neq A\left[\mathfrak{p} t, \mathfrak{p}^{(2)} t^{2}, \mathfrak{p}^{(3)} t^{3}\right]$.

Proof. We will show that $\mathfrak{p}^{(4)} \neq\left[\mathfrak{p}^{(2)}\right]^{2}+\mathfrak{p p}^{(3)}$. Assume the contrary and we have $\ell_{A}\left(A /(Z)+\mathfrak{p}^{(4)}\right)=\ell_{A}\left(A /(Z)+\left[\mathfrak{p}^{(2)}\right]^{2}+\mathfrak{p p}^{(3)}\right)$. Notice that

$$
\begin{aligned}
\ell_{A}\left(A /(Z)+\left[\mathfrak{p}^{(2)}\right]^{2}+\mathfrak{p p}^{(3)}\right) & =10 \alpha^{\prime} \beta+10 \alpha^{\prime} \beta^{\prime}+11 \alpha \beta^{\prime}-2 \alpha \beta & & \text { if } \beta^{\prime}<3 \beta, \\
& =10 \alpha^{\prime} \beta+10 \alpha \beta^{\prime}+10 \alpha^{\prime} \beta^{\prime}-\alpha \beta & & \text { if } \beta^{\prime} \geq 3 \beta
\end{aligned}
$$

by (2.2) and (5.4). Then since $\ell_{A}\left(A /(Z)+\mathfrak{p}^{(4)}\right)=10 \alpha^{\prime} \beta+10 \alpha^{\prime} \beta^{\prime}+10 \alpha \beta^{\prime}$, we get

$$
\begin{aligned}
10 \alpha^{\prime} \beta+10 \alpha^{\prime} \beta^{\prime}+10 \alpha \beta^{\prime} & =10 \alpha^{\prime} \beta+10 \alpha^{\prime} \beta^{\prime}+11 \alpha \beta^{\prime}-2 \alpha \beta & & \text { if } \beta^{\prime}<3 \beta, \\
& =10 \alpha^{\prime} \beta+10 \alpha \beta^{\prime}+10 \alpha^{\prime} \beta^{\prime}-\alpha \beta & & \text { if } \beta^{\prime} \geq 3 \beta .
\end{aligned}
$$

Hence $2 \beta=\beta^{\prime}$, which contradicts our assumption that $2 \beta<\beta^{\prime}$.

The proof of the next proposition is quite similar as that of (6.3) so that we would like to leave it to the readers.

Proposition (6.5). Suppose that $M$ is of type (I) and satisfies $2 \beta \geq \beta^{\prime}$ and $2 \gamma<\gamma^{\prime}$. Then $\mathfrak{p}^{(4)}=\left[\mathfrak{p}^{(2)}\right]^{2}+\mathfrak{p p}^{(3)}$ and the following conditions are equivalent.
(1) $R_{s}(\mathfrak{p})=A\left[\mathfrak{p} t, \mathfrak{p}^{(2)} t^{2}, \mathfrak{p}^{(3)} t^{3}\right]$.
(2) $\mathfrak{p}^{(5)}=\mathfrak{p}^{(2)} \mathfrak{p}^{(3)}$.
(3) $2 \beta=\beta^{\prime}$ or $\beta=\beta^{\prime}$.

When this is the case, $R_{s}(\mathfrak{p})$ is a Gorenstein ring.

Proposition (6.6). Suppose that $M$ is of type (I) and satisfies $2 \alpha \geq \alpha^{\prime}$, $2 \beta<\beta^{\prime}$ and $2 \gamma \geq \gamma^{\prime}$. Then the following conditions are equivalent.

(1) $R_{s}(\mathfrak{p})=A\left[\mathfrak{p} t, \mathfrak{p}^{(2)} t^{2}, \mathfrak{p}^{(3)} t^{3}\right]$.

(2) $\mathfrak{p}^{(4)}=\left[\mathfrak{p}^{(2)}\right]^{2}+\mathfrak{p} \mathfrak{p}^{(3)}$ and $\mathfrak{p}^{(5)}=\mathfrak{p}^{(2)} \mathfrak{p}^{(3)}+\mathfrak{p} \mathfrak{p}^{(4)}$.

(3) $M$ satisfies one of the following conditions:

(i) $\alpha=\alpha^{\prime}$ and $\gamma=\gamma^{\prime}$.

(ii) $2 \alpha=\alpha^{\prime}$ and $\gamma=\gamma^{\prime}$.

When this is the case, $R_{s}(\mathfrak{p})$ is a Gorenstein ring.

Proof. (1) $\Rightarrow(2)$ This is obvious.

(2) $\Rightarrow$ (3) Since $\mathfrak{p}^{(4)}=\left[\mathfrak{p}^{(2)}\right]^{2}+\mathfrak{p} \mathfrak{p}^{(3)}$, we get by (2.2) and (5.4) that

$$
\begin{aligned}
(Y)+\mathfrak{p}^{(4)}= & (Y)+\left(Z^{2 \gamma+4 \gamma^{\prime}}, X^{\alpha} Z^{4 \gamma+2 \gamma^{\prime}}, X^{3 \alpha-\alpha^{\prime}} Z^{2 \gamma+3 \gamma^{\prime}}, X^{2 \alpha} Z^{3 \gamma+2 \gamma^{\prime}},\right. \\
& X^{3 \alpha} Z^{4 \gamma+\gamma^{\prime}}, X^{3 \alpha} Z^{\gamma+3 \gamma^{\prime}}, X^{2 \alpha+\alpha^{\prime}} Z^{2 \gamma+2 \gamma^{\prime}}, X^{4 \alpha} Z^{4 \gamma}, X^{2 \alpha+2 \alpha^{\prime}} Z^{\gamma+2 \gamma^{\prime}}, \\
& \left.X^{4 \alpha+\alpha^{\prime}} Z^{3 \gamma}, X^{4 \alpha+2 \alpha^{\prime}} Z^{2 \gamma}, X^{4 \alpha+3 \alpha^{\prime}} Z^{\gamma}, X^{4 \alpha+4 \alpha^{\prime}}\right) .
\end{aligned}
$$


where we can delete

$$
\begin{aligned}
& X^{3 \alpha} Z^{\gamma+3 \gamma^{\prime}} \quad \text { and } \quad X^{2 \alpha+2 \alpha^{\prime}} Z^{\gamma+2 \gamma^{\prime}} \quad \text { if } 3 \gamma<2 \gamma^{\prime} \text {, } \\
& X^{3 \alpha} Z^{4 \gamma+\gamma^{\prime}} \quad \text { if } 3 \gamma \geq 2 \gamma^{\prime} \text {, }
\end{aligned}
$$

out of the system of generators for the ideal $(Y)+\mathfrak{p}^{(4)}$. Hence

$$
\begin{aligned}
\ell_{A}\left(A /(Y)+\mathfrak{p}^{(4)}\right) & =11 \alpha \gamma+10 \alpha \gamma^{\prime}+10 \alpha^{\prime} \gamma & & \text { if } 3 \gamma<2 \gamma^{\prime}, \\
& =8 \alpha \gamma+12 \alpha \gamma^{\prime}+10 \alpha^{\prime} \gamma & & \text { if } 3 \gamma \geq 2 \gamma^{\prime} .
\end{aligned}
$$

On the other hand, as $\ell_{A}\left(A /(Y)+\mathfrak{p}^{(4)}\right)=e_{Y A}\left(A / \mathfrak{p}^{(4)}\right)=10\left(\alpha \gamma+\alpha \gamma^{\prime}+\alpha^{\prime} \gamma\right)$, we get

$$
\begin{aligned}
10\left(\alpha \gamma+\alpha \gamma^{\prime}+\alpha^{\prime} \gamma\right) & =11 \alpha \gamma+10 \alpha \gamma^{\prime}+10 \alpha^{\prime} \gamma & & \text { if } 3 \gamma<2 \gamma^{\prime} \\
& =8 \alpha \gamma+12 \alpha \gamma^{\prime}+10 \alpha^{\prime} \gamma & & \text { if } 3 \gamma \geq 2 \gamma^{\prime}
\end{aligned}
$$

Hence $\gamma=\gamma^{\prime}$. Thus by (2.2), (5.4) and (\#) we have

$$
\begin{aligned}
(Y)+\mathfrak{p}^{(2)} \mathfrak{p}^{(3)}+\mathfrak{p} \mathfrak{p}^{(4)}= & (Y)+\left(Z^{8 \gamma}, X^{2 \alpha-\alpha^{\prime}} Z^{7 \gamma}, X^{4 \alpha-\alpha^{\prime}} Z^{6 \gamma}, X^{\alpha+\alpha^{\prime}} Z^{6 \gamma},\right. \\
& X^{4 \alpha} Z^{5 \gamma}, X^{4 \alpha+\alpha^{\prime}} Z^{4 \gamma}, X^{3 \alpha+3 \alpha^{\prime}} Z^{3 \gamma}, X^{5 \alpha+3 \alpha^{\prime}} Z^{2 \gamma}, \\
& \left.X^{5 \alpha+4 \alpha^{\prime}} Z^{\gamma}, X^{5 \alpha+5 \alpha^{\prime}}\right) .
\end{aligned}
$$

where we can delete

$$
\begin{array}{ll}
X^{4 \alpha-\alpha^{\prime}} Z^{6 r} & \text { if } 3 \alpha \geq 2 \alpha^{\prime}, \\
X^{\alpha+\alpha^{\prime}} Z^{6 r} & \text { if } 3 \alpha<2 \alpha^{\prime},
\end{array}
$$

out of the system of generators for the ideal $(Y)+\mathfrak{p}^{(2)} \mathfrak{p}^{(3)}+\mathfrak{p} \mathfrak{p}^{(4)}$. Therefore we find

$$
\begin{aligned}
\ell_{A}\left(A /(Y)+\mathfrak{p}^{(2)} \mathfrak{p}^{(3)}+\mathfrak{p p}^{(4)}\right) & =29 \alpha \gamma+16 \alpha^{\prime} \gamma & \text { if } 3 \alpha \geq 2 \alpha^{\prime} \\
& =32 \alpha \gamma+14 \alpha^{\prime} \gamma & \text { if } 3 \alpha<2 \alpha^{\prime}
\end{aligned}
$$

Since $\mathfrak{p}^{(5)}=\mathfrak{p}^{(2)} \mathfrak{p}^{(3)}+\mathfrak{p p}^{(4)}$ by our assumption and since $\ell_{A}\left(A /(Y)+\mathfrak{p}^{(5)}\right)=$ $30 \alpha \gamma+15 \alpha^{\prime} \gamma$, we see

$$
\begin{aligned}
30 \alpha \gamma+15 \alpha^{\prime} \gamma & =29 \alpha \gamma+16 \alpha^{\prime} \gamma & & \text { if } 3 \alpha \geq 2 \alpha^{\prime} \\
& =32 \alpha \gamma+14 \alpha^{\prime} \gamma & & \text { if } 3 \alpha<2 \alpha^{\prime}
\end{aligned}
$$

The first (resp. second) equation implies $\alpha=\alpha^{\prime}$ (resp. $2 \alpha=\alpha^{\prime}$ ).

(3) $\Rightarrow(1)$ We may assume the case (ii) (cf, (4.3)). Let $d_{3}$ be the element in (5.4), (2). Then since $\ell_{A}\left(A /\left(Y, d_{3}, b\right)\right)=1 \cdot 3 \cdot \ell_{A}(A /(Y)+\mathfrak{p})$ and since $A /(b)+\mathfrak{p}^{(2)}$ is a Cohen-Macaulay ring, we have by [5, Theorem (1.1)] that the $A$-algebra $R_{s}(\mathfrak{p})$ is a Gorenstein ring generated in degree three. 
Proposition (6.7). Suppose that $M$ is of type (I) and satisfies $2 \alpha>$ $\alpha^{\prime}>\alpha, 2 \beta \geq \beta^{\prime}$ and $2 \gamma \geq \gamma^{\prime}$. Then the following conditions are equivalent.

(1) $R_{s}(\mathfrak{p})=A\left[\mathfrak{p} t, \mathfrak{p}^{(2)} t^{2}, \mathfrak{p}^{(3)} t^{3}\right]$.

(2) $\mathfrak{p}^{(4)}=\left[\mathfrak{p}^{(2)}\right]^{2}+\mathfrak{p} \mathfrak{p}^{(3)}$ and $\mathfrak{p}^{(5)}=\mathfrak{p}^{(2)} \mathfrak{p}^{(3)}+\mathfrak{p} \mathfrak{p}^{(4)}$.

(3) $M$ satisfies one of the following conditions.

(i) $\beta=\beta^{\prime}$.

(ii) $\gamma=\gamma^{\prime}$.

(iii) $2 \beta=\beta^{\prime}$ and $2 \gamma=\gamma^{\prime}$.

When this is the case, $R_{s}(\mathfrak{p})$ is a Gorenstein ring.

Proof. $\quad(1) \Rightarrow(2)$ This is obvious.

$(2) \Rightarrow(3)$ Since $\ell_{A}\left(A /(X)+\mathfrak{p}^{(4)}\right)=\ell_{A}\left(A /(X)+\left[\mathfrak{p}^{(2)}\right]^{2}+\mathfrak{p p} \mathfrak{p}^{(3)}\right)$, we get

$$
\begin{array}{rlrl}
\ell_{A}\left(A /(X)+\mathfrak{p}^{(4)}\right) & =11 \beta \gamma+9 \beta \gamma^{\prime}+11 \beta^{\prime} \gamma^{\prime}-\beta^{\prime} \gamma & & \text { if } 3 \gamma \geq 2 \gamma^{\prime}, \\
& =8 \beta \gamma+11 \beta \gamma^{\prime}+9 \beta^{\prime} \gamma^{\prime}+2 \beta^{\prime} \gamma & \text { if } 3 \gamma<2 \gamma^{\prime},
\end{array}
$$

while $\ell_{A}\left(A /(X)+\mathfrak{p}^{(4)}\right)=e_{X A}\left(A / \mathfrak{p}^{(4)}\right)=10\left(\beta \gamma+\beta \gamma^{\prime}+\beta^{\prime} \gamma^{\prime}\right)$. Hence we have

$$
\begin{array}{rlrl}
10\left(\beta \gamma+\beta \gamma^{\prime}+\beta^{\prime} \gamma^{\prime}\right) & =11 \beta \gamma+9 \beta \gamma^{\prime}+11 \beta^{\prime} \gamma^{\prime}-\beta^{\prime} \gamma & & \text { if } 3 \gamma \geq 2 \gamma^{\prime}, \\
& =8 \beta \gamma+11 \beta \gamma^{\prime}+9 \beta^{\prime} \gamma^{\prime}+2 \beta^{\prime} \gamma & \text { if } 3 \gamma<2 \gamma^{\prime},
\end{array}
$$

where the first (resp. second) equation implies that $\left(\beta^{\prime}-\beta\right)\left(\gamma^{\prime}-\gamma\right)=0$ (resp. $\left.\left(\beta^{\prime}-\beta\right)\left(2 \gamma-\gamma^{\prime}\right)=0\right)$ so that $\beta=\beta^{\prime}$ or $\gamma=\gamma^{\prime}\left(\right.$ resp. $\beta=\beta^{\prime}$ or $2 \gamma=\gamma^{\prime}$ ).

If $2 \gamma=\gamma^{\prime}$, we have

$$
\begin{aligned}
\ell_{A}\left(A /(X)+\mathfrak{p}^{(2)} \mathfrak{p}^{(3)}+\mathfrak{p} \mathfrak{p}^{(4)}\right) & =47 \beta \gamma+29 \beta^{\prime} \gamma & & \text { if } 3 \beta<2 \beta^{\prime}, \\
& =44 \beta \gamma+31 \beta^{\prime} \gamma & & \text { if } 3 \beta \geq 2 \beta^{\prime} .
\end{aligned}
$$

Therefore since $\ell_{A}\left(A /(X)+\mathfrak{p}^{(5)}\right)=\ell_{A}\left(A /(X)+\mathfrak{p}^{(2)} \mathfrak{p}^{(3)}+\mathfrak{p} \mathfrak{p}^{(4)}\right)$ by our assumption and since $\ell_{A}\left(A /(X)+\mathfrak{p}^{(5)}\right)=e_{X A}\left(A / \mathfrak{p}^{(5)}\right)=15\left(3 \beta \gamma+2 \beta^{\prime} \gamma\right)$, we get

$$
\begin{aligned}
15\left(3 \beta \gamma+2 \beta^{\prime} \gamma\right) & =47 \beta \gamma+29 \beta^{\prime} \gamma & & \text { if } 3 \beta<2 \beta^{\prime}, \\
& =4 \beta \gamma+31 \beta^{\prime} \gamma & & \text { if } 3 \beta \geq 2 \beta^{\prime} .
\end{aligned}
$$

The first (resp. second) equation implies $2 \beta=\beta^{\prime}$ (resp. $\beta=\beta^{\prime}$ ), whence the assertion (3).

(3) $\Rightarrow$ (1) We may assume the cases (ii) and (iii) (cf. (4.3)). First assume the case (ii) and let $d_{2}$ and $d_{3}$ be the elements obtained by (2.2) and (2) of (5.4). Then we have

$$
\begin{aligned}
\ell_{A}\left(A /\left(d_{2}, d_{3}, X\right)\right) & =\ell_{A}\left(A /\left(Z^{3 \gamma}, Y^{4 \beta+2 \beta^{\prime}}, X\right)\right) \\
& =3 \gamma\left(4 \beta+2 \beta^{\prime}\right) \\
& =2 \cdot 3 \cdot \ell_{A}(A /(X)+\mathfrak{p}) .
\end{aligned}
$$


Hence $R_{s}(\mathfrak{p})$ is Noetherian (cf. [11, Theorem (3.1)]). We put $I=\left(d_{2}\right)+\mathfrak{p}^{(3)}$. Then since

$$
(X)+I=(X)+\left(Z^{3 \gamma}, Y^{3 \beta+\beta^{\prime}} Z^{2 \gamma}, Y^{3 \beta+2 \beta^{\prime}} Z^{\gamma}, Y^{4 \beta+2 \beta^{\prime}}\right)
$$

by (5.4), we get $\ell_{A}(A /(X)+I)=10 \beta \gamma+5 \beta^{\prime} \gamma=e_{X A}(A / I)$ so that $A / I$ is Cohen-Macaulay. Thus $R_{s}(\mathfrak{p})$ is a Gorenstein ring generated in degree three (cf. [5, Theorem (1.1)]).

Secondly we consider the case (iii). Let $d_{3}^{\prime}$ be the element in (2) of (5.4). Then $\ell_{A}\left(A /\left(d_{3}^{\prime}, c, X\right)\right)=1 \cdot 3 \cdot \ell_{A}(A /(X)+\mathfrak{p})$ and $A /(c)+\mathfrak{p}^{(2)}$ is CohenMacaulay. Hence $R_{s}(\mathfrak{p})$ is a Gorenstein ring generated in degree three.

Proposition (6.8). Suppose that $M$ is of type (I) and satisfies $2 \alpha \geq \alpha^{\prime}$, $2 \beta>\beta^{\prime}>\beta$ and $2 \gamma \geq \gamma^{\prime}$. Then the following conditions are equivalent.

(1) $R_{s}(\mathfrak{p})=A\left[\mathfrak{p} t, \mathfrak{p}^{(2)} t^{2}, \mathfrak{p}^{(3)} t^{3}\right]$.

(2) $\mathfrak{p}^{(4)}=\left[\mathfrak{p}^{(2)}\right]^{2}+\mathfrak{p} \mathfrak{p}^{(3)}$ and $\mathfrak{p}^{(5)}=\mathfrak{p}^{(2)} \mathfrak{p}^{(3)}+\mathfrak{p} \mathfrak{p}^{(4)}$.

(3) $\gamma=\gamma^{\prime}$.

When this is the case, $R_{s}(\mathfrak{p})$ is a Gorenstein ring.

Proof. $\quad(1) \Rightarrow(2)$ This is obvious.

$(2) \Rightarrow(3)$ Similarly as in Proof of (6.7) we get

$$
\begin{aligned}
\ell_{A}\left(A /(Y)+\left[\mathfrak{p}^{(2)}\right]^{2}+\mathfrak{p} \mathfrak{p}^{(3)}\right) & =8 \alpha \gamma+12 \alpha \gamma^{\prime}+11 \alpha^{\prime} \gamma-\alpha^{\prime} \gamma^{\prime} & & \text { if } 3 \gamma \geq 2 \gamma^{\prime}, \\
& =14 \alpha \gamma+8 \alpha \gamma^{\prime}+8 \alpha^{\prime} \gamma+\alpha^{\prime} \gamma^{\prime} & & \text { if } 3 \gamma<2 \gamma^{\prime} .
\end{aligned}
$$

Since $\mathfrak{p}^{(4)}=\left[\mathfrak{p}^{(2)}\right]^{2}+\mathfrak{p} \mathfrak{p}^{(3)}$, we have

$$
\begin{aligned}
10\left(\alpha \gamma+\alpha \gamma^{\prime}+\alpha^{\prime} \gamma\right) & =8 \alpha \gamma+12 \alpha \gamma^{\prime}+11 \alpha^{\prime} \gamma-\alpha^{\prime} \gamma^{\prime} & & \text { if } 3 \gamma \geq 2 \gamma^{\prime}, \\
& =14 \alpha \gamma+8 \alpha \gamma^{\prime}+8 \alpha^{\prime} \gamma+\alpha^{\prime} \gamma^{\prime} & & \text { if } 3 \gamma<2 \gamma^{\prime} .
\end{aligned}
$$

The first (resp. second) equation implies $\left(2 \alpha-\alpha^{\prime}\right)\left(\gamma-\gamma^{\prime}\right)=0$ (resp. $\left(2 \alpha-\alpha^{\prime}\right)$ $\left(2 \gamma-\gamma^{\prime}\right)=0$ ) and so $2 \alpha=\alpha^{\prime}$ or $\gamma=\gamma^{\prime}$ (resp. $2 \alpha=\alpha^{\prime}$ or $\left.2 \gamma=\gamma^{\prime}\right)$. We want to show that the equality $2 \gamma=\gamma^{\prime}$ is impossible and that $\gamma=\gamma^{\prime}$, if $2 \alpha=\alpha^{\prime}$. First assume that $2 \alpha=\alpha^{\prime}$. Then we have

$$
\begin{aligned}
\ell_{A}\left(A /(Y)+\mathfrak{p}^{(2)} \mathfrak{p}^{(3)}+\mathfrak{p} \mathfrak{p}^{(4)}\right) & =43 \alpha \gamma+17 \alpha \gamma^{\prime} & & \text { if } 3 \gamma \geq 2 \gamma^{\prime}, \\
& =46 \alpha \gamma+15 \alpha \gamma^{\prime} & & \text { if } 3 \gamma<2 \gamma^{\prime} .
\end{aligned}
$$

Hence by the assumption that $\mathfrak{p}^{(5)}=\mathfrak{p}^{(2)} \mathfrak{p}^{(3)}+\mathfrak{p} \mathfrak{p}^{(4)}$ we get

$$
\begin{aligned}
45 \alpha \gamma+15 \alpha \gamma^{\prime} & =43 \alpha \gamma+17 \alpha \gamma^{\prime} & & \text { if } 3 \gamma \geq 2 \gamma^{\prime}, \\
& =46 \alpha \gamma+15 \alpha \gamma^{\prime} & & \text { if } 3 \gamma<2 \gamma^{\prime},
\end{aligned}
$$


whence $\gamma=\gamma^{\prime}$. Now assume that $2 \gamma=\gamma^{\prime}$. Then

and so

$$
\ell_{A}\left(A /(Y)+\mathfrak{p}^{(2)} \mathfrak{p}^{(3)}+\mathfrak{p p}^{(4)}\right)=46 \alpha \gamma+15 \alpha^{\prime} \gamma,
$$

$$
45 \alpha \gamma+15 \alpha^{\prime} \gamma=46 \alpha \gamma+15 \alpha^{\prime} \gamma,
$$

which implies $\alpha \gamma=0$. Thus $2 \gamma \neq \gamma^{\prime}$.

(3) $\Rightarrow$ (1) Let $d_{2}$ and $d_{3}$ be the elements obtained by (2.2) and (2) of (5.4). Then $\ell_{A}\left(A /\left(d_{2}, d_{3}, Y\right)\right)=2 \cdot 3 \cdot \ell_{A}(A /(Y)+\mathfrak{p})$ and $A /\left(d_{2}\right)+\mathfrak{p}^{(3)}$ is CohenMacaulay. Therefore $R_{s}(\mathfrak{p})$ is a Gorenstein ring generated in degree three by [5, Theorem (1.1)].

Proposition (6.9). Suppose that $M$ is of type (I) and satisfies $2 \alpha \geq \alpha^{\prime}$, $2 \beta \geq \beta^{\prime}$ and $2 \gamma>\gamma^{\prime}>\gamma$. Then the following conditions are equivalent.

(1) $R_{s}(\mathfrak{p})=A\left[\mathfrak{p} t, \mathfrak{p}^{(2)} t^{2}, \mathfrak{p}^{(3)} t^{3}\right]$.

(2) $\mathfrak{p}^{(4)}=\left[\mathfrak{p}^{(2)}\right]^{2}+\mathfrak{p} \mathfrak{p}^{(3)}$ and $\mathfrak{p}^{(5)}=\mathfrak{p}^{(2)} \mathfrak{p}^{(3)}+\mathfrak{p} \mathfrak{p}^{(4)}$.

(3) $M$ satisfies one of the following conditions.

(i) $\beta=\beta^{\prime}$.

(ii) $2 \alpha=\alpha^{\prime}$ and $2 \beta=\beta^{\prime}$.

When this is the case, $R_{s}(\mathfrak{p})$ is a Gorenstein ring.

Proof. $\quad(1) \Rightarrow(2)$ This is obvious.

$(2) \Rightarrow(3) \quad A s \quad \ell_{A}\left(A /(Z)+\mathfrak{p}^{(4)}\right)=\ell_{A}\left(A /(Z)+\left[\mathfrak{p}^{(2)}\right]^{2}+\mathfrak{p p}^{(3)}\right)$, we get

$$
10\left(\alpha^{\prime} \beta+\alpha^{\prime} \beta^{\prime}+\alpha \beta^{\prime}\right)=11 \alpha^{\prime} \beta+9 \alpha^{\prime} \beta^{\prime}+12 \alpha \beta^{\prime}-2 \alpha \beta,
$$

whence $\left(2 \alpha-\alpha^{\prime}\right)\left(\beta-\beta^{\prime}\right)=0$. Thus $2 \alpha=\alpha^{\prime}$ or $\beta=\beta^{\prime}$. Assume $2 \alpha=\alpha^{\prime}$. Then since $\mathfrak{p}^{(5)}=\mathfrak{p}^{(2)} \mathfrak{p}^{(3)}+\mathfrak{p} \mathfrak{p}^{(4)}$, we have

$$
\begin{aligned}
30 \alpha \beta+45 \alpha \beta^{\prime} & =29 \alpha \beta+46 \alpha \beta^{\prime} & & \text { if } 3 \beta \geq 2 \beta^{\prime}, \\
& =32 \alpha \beta+44 \alpha \beta^{\prime} & & \text { if } 3 \beta<2 \beta^{\prime} .
\end{aligned}
$$

The first (resp. second) equation induces $\beta=\beta^{\prime}$ (resp. $2 \beta=\beta^{\prime}$ ) as required.

$(3) \Rightarrow(1)$ We may assume the case (ii). Let $d_{3}$ be the element in (2) of (5.4). Then $\ell_{A}\left(A /\left(d_{3}, c, Z\right)\right)=1 \cdot 3 \cdot \ell_{A}(A /(Z)+\mathfrak{p})$ and $A /(c)+\mathfrak{p}^{(2)}$ is CohenMacaulay. Hence $R_{s}(\mathfrak{p})$ is a Gorenstein ring generated in degree three (cf. [5, Theorem (1.1)]).

The next assertion is similarly proved as the implication $(3) \Rightarrow(1)$ in (6.9).

Proposition (6.10). Suppose that $M$ is of type (I) and satisfies $2 \alpha=\alpha^{\prime}$ and $2 \beta=\beta^{\prime}$. Then $R_{s}(\mathfrak{p})=A\left[\mathfrak{p} t, \mathfrak{p}^{(2)} t^{2}, \mathfrak{p}^{(3)} t^{3}\right]$ and it is a Gorenstein ring. 
Proposition (6.11). Suppose that $M$ is of type (I) and satisfies $\alpha=\alpha^{\prime}$, $2 \beta=\beta^{\prime}$ and $2 \gamma=\gamma^{\prime}$. Then $R_{s}(\mathfrak{p})=A\left[\mathfrak{p} t, \mathfrak{p}^{(2)} t^{2}, \mathfrak{p}^{(3)} t^{3}\right]$ and it is a Gorenstein ring.

Proof. Let $d_{3}^{\prime}$ be the element in (2) of (5.4). Then $\ell_{A}\left(A /\left(d_{3}^{\prime}, c, Y\right)\right)=$ $1 \cdot 3 \cdot \ell_{A}(A /(Y)+\mathfrak{p})$ and $A /(c)+\mathfrak{p}^{(2)}$ is Cohen-Macaulay. Hence $R_{s}(\mathfrak{p})$ is a Gorenstein ring generated in degree three.

For the rest of this section we assume that $A$ contains a field $k$ and that $M$ has type (II). The purpose is to prove the following

Theorem (6.12). Suppose that $M$ has type (II) and satisfies the condition

(\#) $\alpha \neq 2 \alpha^{\prime}, 2 \beta \neq \beta^{\prime}$ or $2 \gamma \neq \gamma^{\prime}$.

Then the following assertions are equivalent.

(1) $R_{s}(\mathfrak{p})=A\left[\mathfrak{p} t, \mathfrak{p}^{(2)} t^{2}, \mathfrak{p}^{(3)} t^{3}\right]$.

(2) The characteristic of $k$ is equal to 2 and $M$ satisfies one of the following conditions.

$$
\begin{aligned}
& \text { (i) } 2 \alpha^{\prime} \leq \alpha, 2 \beta \geq \beta^{\prime} \text { and } 2 \gamma=\gamma^{\prime} \text {. } \\
& \text { (ii) } 2 \alpha^{\prime} \geq \alpha, 2 \beta=\beta^{\prime} \text { and } 2 \gamma \leq \gamma^{\prime} \text {. } \\
& \text { (iii) } 2 \alpha^{\prime}=\alpha, 2 \beta \leq \beta^{\prime} \text { and } 2 \gamma \geq \gamma^{\prime} \text {. }
\end{aligned}
$$

When this is the case, $R_{s}(\mathfrak{p})$ is not a Cohen-Macaulay ring.

We divide the proof of Theorem (6.12) into the cases stated in (5.10). The proof will be completed by (6.16), summarizing the results of (6.13), (6.14), (6.15) and (6.16).

We begin with the following

Proposition (6.13). Suppose that $M$ has type (II) and satisfies $2 \alpha^{\prime}<\alpha$, $2 \beta<\beta^{\prime}$ and $2 \gamma<\gamma^{\prime}$. Then $\left[\mathfrak{p}^{(2)}\right]^{2}+\mathfrak{p} \mathfrak{p}^{(3)} \neq \mathfrak{p}^{(4)}$.

Proof. First assume that $\operatorname{ch} k \neq 2$. Since by (2.3) and (5.10) we explicitly have a system of generators for the ideal $(X)+\left[\mathfrak{p}^{(2)}\right]^{2}+\mathfrak{p p}^{(3)}$, we get

$$
\begin{aligned}
\ell_{A}\left(A /(X)+\left[\mathfrak{p}^{(2)}\right]^{2}+\mathfrak{p p ^ { ( 3 ) } )}\right. & =16 \beta \gamma+10 \beta \gamma^{\prime}+10 \beta^{\prime} \gamma^{\prime} \quad \text { if } 3 \beta \leq \beta^{\prime} \text { and } 3 \gamma \leq \gamma^{\prime}, \\
& =7 \beta \gamma+13 \beta \gamma^{\prime}+10 \beta^{\prime} \gamma^{\prime} \quad \text { if } 3 \beta \leq \beta^{\prime} \text { and } 3 \gamma>\gamma^{\prime}, \\
& =7 \beta \gamma+10 \beta \gamma^{\prime}+10 \beta^{\prime} \gamma^{\prime}+3 \beta^{\prime} \gamma \\
&
\end{aligned}
$$

Therefore if $\mathfrak{p}^{(4)}=\left[\mathfrak{p}^{(2)}\right]^{2}+\mathfrak{p p}^{(3)}$, then since $\ell_{A}\left(A /(X)+\mathfrak{p}^{(4)}\right)=10\left(\beta \gamma+\beta \gamma^{\prime}\right.$ 
$\left.+\beta^{\prime} \gamma^{\prime}\right)$, we have by the above equalities that

$$
\begin{array}{ll}
\beta \gamma=0 & \text { if } 3 \beta \leq \beta^{\prime} \text { and } 3 \gamma \leq \gamma^{\prime}, \\
\gamma=\gamma^{\prime} & \text { if } 3 \beta \leq \beta^{\prime} \text { and } 3 \gamma>\gamma^{\prime}, \\
\beta=\beta^{\prime} & \text { if } 3 \beta>\beta^{\prime} \text { and } 3 \gamma \leq \gamma^{\prime} \text { and } \\
3 \beta \gamma=\beta^{\prime} \gamma^{\prime}>4 \beta \gamma & \text { if } 3 \beta>\beta^{\prime} \text { and } 3 \gamma>\gamma^{\prime},
\end{array}
$$

all of which are impossible. Hence $\mathfrak{p}^{(4)} \neq\left[\mathfrak{p}^{(2)}\right]^{2}+\mathfrak{p p}^{(3)}$.

Secondly assume that $\operatorname{ch} k=2$ and that $\left[\mathfrak{p}^{(2)}\right]^{2}+\mathfrak{p p}^{(3)}=\mathfrak{p}^{(4)}$. Then by (2.3) and (5.10) we get

$$
\begin{aligned}
10\left(\beta \gamma+\beta \gamma^{\prime}+\beta^{\prime} \gamma^{\prime}\right) & =13 \beta \gamma+10 \beta \gamma^{\prime}+10 \beta^{\prime} \gamma^{\prime} & & \text { if } 3 \beta \leq \beta^{\prime} \text { and } 3 \gamma \leq \gamma^{\prime}, \\
& =7 \beta \gamma+12 \beta \gamma^{\prime}+10 \beta^{\prime} \gamma^{\prime} & & \text { if } 3 \beta \leq \beta^{\prime} \text { and } 3 \gamma>\gamma^{\prime}, \\
& =7 \beta \gamma+10 \beta \gamma^{\prime}+10 \beta^{\prime} \gamma^{\prime}+2 \beta^{\prime} \gamma & & \text { if } 3 \beta>\beta^{\prime} \text { and } 3 \gamma \leq \gamma^{\prime}, \\
& =10 \beta \gamma+9 \beta \gamma^{\prime}+11 \beta^{\prime} \gamma^{\prime}-\beta^{\prime} \gamma & & \text { if } 3 \beta>\beta^{\prime} \text { and } 3 \gamma>\gamma^{\prime},
\end{aligned}
$$

all of which contradict the standard assumption on $\beta$ and $\gamma$. Hence $\mathfrak{p}^{(4)} \neq\left[\mathfrak{p}^{(2)}\right]^{2}+\mathfrak{p p}^{(3)}$.

Proposition (6.14). Suppose that $M$ has type (II) and satisfies $2 \alpha^{\prime}<\alpha$, $2 \beta<\beta^{\prime}$ and $2 \gamma \geq \gamma^{\prime}$. Then $\left[\mathfrak{p}^{(2)}\right]^{2}+\mathfrak{p p}^{(3)} \neq \mathfrak{p}^{(4)}$.

Proof. Assume that $\mathfrak{p}^{(4)}=\left[\mathfrak{p}^{(2)}\right]^{2}+\mathfrak{p p}^{(3)}$. Then if $\operatorname{ch} k \neq 2$, we have

$$
\begin{aligned}
10\left(\beta \gamma+\beta \gamma^{\prime}+\beta^{\prime} \gamma^{\prime}\right) & =9 \beta \gamma+12 \beta \gamma^{\prime}+10 \beta^{\prime} \gamma^{\prime} & & \text { if } 3 \beta \leq \beta^{\prime}, \\
& =15 \beta \gamma+6 \beta \gamma^{\prime}+12 \beta^{\prime} \gamma^{\prime}-2 \beta^{\prime} \gamma & & \text { if } 3 \beta>\beta^{\prime},
\end{aligned}
$$

which contradict the assumption on $\beta$ and $\gamma$. Therefore the characteristic of $k$ has to be 2. But when $\operatorname{ch} k=2$, we have the equalities

$$
\begin{aligned}
10\left(\beta \gamma+\beta \gamma^{\prime}+\beta^{\prime} \gamma^{\prime}\right) & =11 \beta \gamma+10 \beta \gamma^{\prime}+10 \beta^{\prime} \gamma^{\prime} & & \text { if } 3 \beta \leq \beta^{\prime}, \\
& =14 \beta \gamma+7 \beta \gamma^{\prime}+11 \beta^{\prime} \gamma^{\prime}-\beta^{\prime} \gamma & & \text { if } 3 \beta>\beta^{\prime},
\end{aligned}
$$

both of which again contradict the assumption on $\beta$ and $\gamma$, too. Thus we have $\mathfrak{p}^{(4)} \neq\left[\mathfrak{p}^{(2)}\right]^{2}+\mathfrak{p} \mathfrak{p}^{(3)}$.

Proposition (6.15). Suppose that $M$ has type (II) and satisfies $2 \alpha^{\prime}<\alpha$, $2 \beta \geq \beta^{\prime}$ and $2 \gamma \geq \gamma^{\prime}$. Then

(1) $\left[\mathfrak{p}^{(2)}\right]^{2}+\mathfrak{p p}^{(3)} \neq \mathfrak{p}^{(4)}$ if $\operatorname{ch} k \neq 2$.

(2) Suppose that $\operatorname{ch} k=2$. Then $R_{s}(\mathfrak{p})=A\left[\mathfrak{p} t, \mathfrak{p}^{(2)} t^{2}, \mathfrak{p}^{(3)} t^{3}\right]$ if and only if $2 \gamma=\gamma^{\prime}$. When this is the case, $R_{s}(\mathfrak{p})$ is not a Cohen-Macaulay ring.

Proof. (1) Assume that $\mathfrak{p}^{(4)}=\left[\mathfrak{p}^{(2)}\right]^{2}+\mathfrak{p p}^{(3)}$. Then we have 


$$
\begin{aligned}
10\left(\beta \gamma+\beta \gamma^{\prime}+\beta^{\prime} \gamma^{\prime}\right) & =13 \beta \gamma+8 \beta \gamma^{\prime}+11 \beta^{\prime} \gamma^{\prime}-\beta^{\prime} \gamma & & \text { if } 3 \beta<2 \beta^{\prime}, \\
& =7 \beta \gamma+11 \beta \gamma^{\prime}+9 \beta^{\prime} \gamma^{\prime}+3 \beta^{\prime} \gamma & & \text { if } 3 \beta \geq 2 \beta^{\prime} .
\end{aligned}
$$

Both the equations contradict the assumption on $\beta$ and $\gamma$, whence $\mathfrak{p}^{(4)} \neq$ $\left[\mathfrak{p}^{(2)}\right]^{2}+\mathfrak{p p}^{(3)}$.

(2) Suppose that $R_{s}(\mathfrak{p})=A\left[\mathfrak{p} t, \mathfrak{p}^{(2)} t^{2}, \mathfrak{p}^{(3)} t^{3}\right]$. Then $\mathfrak{p}^{(4)}=\left[\mathfrak{p}^{(2)}\right]^{2}+\mathfrak{p} \mathfrak{p}^{(3)}$ so that

$$
\begin{aligned}
10\left(\beta \gamma+\beta \gamma^{\prime}+\beta^{\prime} \gamma^{\prime}\right) & =12 \beta \gamma+9 \beta \gamma^{\prime}+10 \beta^{\prime} \gamma^{\prime} & & \text { if } 3 \beta<2 \beta^{\prime} . \\
& =6 \beta \gamma+12 \beta \gamma^{\prime}+8 \beta^{\prime} \gamma^{\prime}+4 \beta^{\prime} \gamma & & \text { if } 3 \beta \geq 2 \beta^{\prime},
\end{aligned}
$$

whence we have $2 \gamma=\gamma^{\prime}$. Conversely assume that $2 \gamma=\gamma^{\prime}$ and let $e_{3}$ be the element in (2) of (5.10). Then since

$$
\begin{aligned}
\ell_{A}\left(A /\left(e_{3}, a, X\right)\right) & =\ell_{A}\left(A /\left(Y^{3 \beta+2 \beta^{\prime}}, Z^{3 \gamma}, X\right)\right) \\
& =3 \gamma\left(3 \beta+2 \beta^{\prime}\right) \\
& =1 \cdot 3 \cdot \ell_{A}(A /(X)+\mathfrak{p}),
\end{aligned}
$$

we get $R_{s}(\mathfrak{p})=A\left[\mathfrak{p} t, d_{2} t^{2}, e_{3} t^{3}\right]$ by [5, Corollary (3.8)]. The ring $R_{s}(\mathfrak{p})$ is not Cohen-Macaulay (cf. [5, Theorem (1.1)] and [20, Corollary (3.4)]), because $A /(a)+\mathfrak{p}^{(2)}$ is non-Cohen-Macaulay by [15, Proposition (2.4)].

Proposition (6.16). Suppose that $M$ has type (II) and satisfies $2 \alpha^{\prime}>\alpha$, $2 \beta \geq \beta^{\prime}$ and $2 \gamma \geq \gamma^{\prime}$. Then the following assertions hold.

(1) If $\operatorname{ch} k \neq 2$, then $\left[\mathfrak{p}^{(2)}\right]^{2}+\mathfrak{p} \mathfrak{p}^{(3)} \neq \mathfrak{p}^{(4)}$ or $\mathfrak{p}^{(2)} \mathfrak{p}^{(3)}+\mathfrak{p} \mathfrak{p}^{(4)} \neq \mathfrak{p}^{(5)}$.

(2) Suppose that $\operatorname{ch} k=2$. Then $R_{s}(\mathfrak{p})=A\left[\mathfrak{p} t, \mathfrak{p}^{(2)} t^{2}, \mathfrak{p}^{(3)} t^{3}\right]$ if and only if $2 \beta=\beta^{\prime}$ and $2 \gamma=\gamma^{\prime}$. When this is the case, $R_{s}(\mathfrak{p})$ is not Cohen-Macaulay.

Proof. (1) Assume $\mathfrak{p}^{(4)}=\left[\mathfrak{p}^{(2)}\right]^{2}+\mathfrak{p p}^{(3)}$ and we will prove that $\mathfrak{p}^{(2)} \mathfrak{p}^{(3)}$ $+\mathfrak{p p}^{(4)} \neq \mathfrak{p}^{(5)}$. By the equality $\ell_{A}\left(A /(X)+\mathfrak{p}^{(4)}\right)=\ell_{A}\left(A /(X)+\left[\mathfrak{p}^{(2)}\right]^{2}+\mathfrak{p p}^{(3)}\right)$ we get that

$$
\begin{aligned}
10\left(\beta \gamma+\beta \gamma^{\prime}+\beta^{\prime} \gamma^{\prime}\right)= & 15 \beta \gamma+7 \beta \gamma^{\prime}+12 \beta^{\prime} \gamma^{\prime}-3 \beta^{\prime} \gamma \\
& \quad \text { if } 3 \beta<2 \beta^{\prime} \text { and } 3 \gamma \geq 2 \gamma^{\prime}, \\
= & 6 \beta \gamma+13 \beta \gamma^{\prime}+8 \beta^{\prime} \gamma^{\prime}+3 \beta^{\prime} \gamma \\
& \text { if (i) } 3 \beta \geq 2 \beta^{\prime} \text { or if (ii) } 3 \beta<2 \beta^{\prime} \text { and } 3 \gamma<2 \gamma^{\prime} .
\end{aligned}
$$

By these equations we find $2 \beta=\beta^{\prime}$ and $2 \gamma=\gamma^{\prime}$, whence $\ell_{A}\left(A /(X)+\mathfrak{p}^{(2)} \mathfrak{p}^{(3)}\right.$ $\left.+\mathfrak{p p}^{(4)}\right)=108 \beta \gamma$ and $\ell_{A}\left(A /(X)+\mathfrak{p}^{(5)}\right)=105 \beta \gamma$. Thus $\mathfrak{p}^{(2)} \mathfrak{p}^{(3)}+\mathfrak{p} \mathfrak{p}^{(4)} \neq \mathfrak{p}^{(5)}$ as required.

(2) Suppose that $R_{s}(\mathfrak{p})=A\left[\mathfrak{p} t, \mathfrak{p}^{(2)} t^{2}, \mathfrak{p}^{(3)} t^{3}\right]$. Then $\mathfrak{p}^{(4)}=\left[\mathfrak{p}^{(2)}\right]^{2}+\mathfrak{p} \mathfrak{p}^{(3)}$, 
which yields the same equations as $(\sharp)$ above so that we have $2 \beta=\beta^{\prime}$ and $2 \gamma=\gamma^{\prime}$. Conversely assume $2 \beta=\beta^{\prime}$ and $2 \gamma=\gamma^{\prime}$ and let $e_{3}$ be the element obtained by (2) of (5.6). Then since

$$
\begin{aligned}
\ell_{A}\left(A /\left(e_{3}, c, X\right)\right) & =\ell_{A}\left(A /\left(Z^{7 r}, Y^{3 \beta}, X\right)\right) \\
& =21 \beta \gamma \\
& =3 \cdot \ell_{A}(A /(X)+\mathfrak{p}),
\end{aligned}
$$

we get $R_{s}(\mathfrak{p})=A\left[\mathfrak{p} t, d_{2} t^{2}, e_{3} t^{3}\right]$ by $\left[5\right.$, Corollary (3.8)]. As $A /(c)+\mathfrak{p}^{(2)}$ is non-Cohen-Macaulay by [15, Proposition (2.4)], the $A$-algebra $R_{s}(\mathfrak{p})$ cannot be Cohen-Macaulay (cf. [5, Theorem (1.1)] and [20, Corollary (3.4)]).

EXAmple (6.17). (1) Let $\mathfrak{p}=\mathfrak{p}(10,11,13)$ (resp. $\mathfrak{p}(11,16,13)$ ). Then $\mathfrak{p}$ is generated by the maximal minors of the matrix

$$
\left[\begin{array}{lll}
X^{2} & Y^{2} & Z^{3} \\
Y & Z & X^{3}
\end{array}\right] \quad\left(\operatorname{resp} .\left[\begin{array}{lll}
X^{2} & Y^{2} & Z^{3} \\
Y & Z^{2} & X^{3}
\end{array}\right]\right)
$$

Hence by (6.1) $R_{s}(\mathfrak{p})$ is generated (resp. not generated) in degree three.

(2) Suppose that $\operatorname{ch} k=2$ and let $\mathfrak{p}=\mathfrak{p}(17,10,19)$ (resp. $\mathfrak{p}(7,11,12)$ ). Then $\mathfrak{p}$ is generated by the maximal minors of the matrix

$$
\left[\begin{array}{lll}
X^{3} & Y^{4} & Z^{2} \\
Y^{3} & Z & X
\end{array}\right] \quad\left(\text { resp. }\left[\begin{array}{lll}
X^{3} & Y^{2} & Z^{2} \\
Y & Z & X^{2}
\end{array}\right]\right)
$$

and so $R_{s}(\mathfrak{p})$ is generated in degree three by (6.16) (resp. (6.17)) but not Cohen-Macaulay.

\section{§7. A note on Huckaba's theorem on the analytic spread modulo one element}

Let $A$ be an unmixed Noetherian local ring and $\mathfrak{p}$ a prime ideal in $A$ of $\operatorname{dim} A / \mathfrak{p}=1$. Then S. Huckaba [10] discussed the behavior of the analytic spread $\lambda(\mathfrak{p})$ of $\mathfrak{p}$ modulo one element and gave, in terms of $\lambda(\mathfrak{p})$, a sufficient condition for $R_{s}(\mathfrak{p})$ to be Noetherian. In this section we shall try to improve his sufficient condition in the case where $A$ is a regular local ring of $\operatorname{dim} A=3$, clarifying the relationship between Huckaba's condition and Huneke's criterion [13, Theorem 1.4] of $R_{s}(\mathfrak{p})$ being Noetherian. Our improvement below also provides a partial answer to the question posed by Huckaba [10, Remark 2.6, (3)]:

Theorem (7.1). Let $A$ be a 3-dimensional regular local ring with maximal ideal $\mathfrak{m}$ and $\mathfrak{p}$ a prime ideal in $A$ of $\operatorname{dim} A / \mathfrak{p}=1$. Let $0 \neq g \in \mathfrak{p}$ 
and let $\ell$ be the largest integer with $g \in \mathfrak{p}^{(\ell)}$. Assume that the field $A / \mathfrak{m}$ is infinite. Then the following two conditions are equivalent.

(1) $\lambda(\mathfrak{p} / g A)=1$.

(2) $\ell_{A}(A /(f, g, x))=\ell \cdot \ell_{A}(A /(X)+\mathfrak{p})$ for some $f \in \mathfrak{p}$ and $x \in \mathfrak{m} \backslash \mathfrak{p}$.

When this is the case, $R_{s}(\mathfrak{p})=A\left[\mathfrak{p}^{(n)} t^{n} \mid 1 \leq n \leq \ell\right]$.

Proof. (1) $\Rightarrow(2) \quad$ We put $B=A_{\mathfrak{p}}$ and $\mathfrak{n}=\mathfrak{p} A_{\mathfrak{p}}$. Choose $f \in \mathfrak{p}$ so that $\mathfrak{p}^{r+1}+g A=f \mathfrak{p}^{r}+g A$ for some $r \geq 0$. Then $\mathfrak{p}=\sqrt{(f, g)}$ and $\mathfrak{n}^{r+1}+g B=$ $f \mathfrak{n}^{r}+g B$. Hence

$$
\begin{aligned}
\mathfrak{n}^{r+1} & =f \mathfrak{n}^{r}+g B \cap \mathfrak{n}^{r+1} \\
& =f \mathfrak{n}^{r}+g \mathfrak{n}^{r+1-\ell}
\end{aligned}
$$

so that $f, g$ forms a super regular sequence in $B$. Since $\ell_{B}(B /(f, g) B)=\ell$, we get by the additive formula $[18$, p. 126] of multiplicity the required equality that

$$
\begin{aligned}
\ell_{A}(A /(f, g, x)) & =e_{X A}(A /(f, g)) \\
& =\ell_{B}(B /(f, g) B) \cdot e_{X A}(A / \mathfrak{p}) \\
& =\ell \cdot \ell_{A}(A /(x)+\mathfrak{p})
\end{aligned}
$$

for any $x \in \mathfrak{m} \backslash p$.

(2) $\Rightarrow(1)$ We put $C=A / g A$ and $P=p / g A$. Then since $A / g A+\mathfrak{p}^{(n)}$ is a Cohen-Macaulay ring for $n \geq 1$ and since $\mathfrak{p}^{(n)}=f \mathfrak{p}^{(n-1)}+g \mathfrak{p}^{(n-\ell)}$ for $n \geq \ell$ (cf. [5, (3.7) (1)], we have that $P^{(n)}=\mathfrak{p}^{(n)} C$ for $n \geq 1$ and that $P^{(n)}$ $=P P^{(n-1)}$ for all $n \geq \ell$. Therefore $R_{s}(P)$ is module-finite over $R(P)=$ $\sum_{n \geq 0} P^{n} t^{n}$ and so we get by [4, Theorem (2.10)] that

$$
\lambda(P \hat{C}+Q / Q)<\operatorname{dim} \hat{C} / Q=2
$$

for any $Q \in$ Ass $\hat{C}$, where $\hat{C}$ denotes the $\mathrm{m} / g A$-adic completion of $C$. Thus we have $\lambda(P)=1$, because

$$
\lambda(P)=\lambda(P \hat{C})=\lambda(P \hat{C}+Q / Q)
$$

for some $Q \in$ Ass $\hat{C}$ (cf. [14, Lemma 4.2]). The last assertion directly follows from [5, Corollary (3.8)]. 


\section{REFERENCES}

[1] Buchsbaum, D. A. and Eisenbud, D., What makes a complex exact?, J. Alg., 25 (1973), 259-268.

[2] - Algebra structures for finite free resolutions and structure theorems for ideals of codimension 3, Amer. J. Math., 99 (1977), 447-485.

[ 3 ] Burch, L., Codimension and analytic spread, Proc. Camb. Phil. Soc., 72 (1972), 369-373.

[4] Goto, S., Herrmann, M., Nishida, K. and Villamayor, O., On the structure of Noetherian symbolic Rees algebras, manuscripta math., 67 (1990), 197-225.

[ 5 ] Goto, S., Nishida, K. and Shimoda, Y., The Gorensteinness of symbolic Rees algebras for space curves, J. Math. Soc. Japan, 43 (1991), 465-481.

[6] — The Gorensteinness of the symbolic blow-ups for certain space monomial curves, to appear in Trans. Amer. Math. Soc.

[ 7 ] Herzog, J., Generators and relations of abelian semigroups and semigroup rings, manuscripta math., 3 (1970), 175-193.

[ 8 ] Herzog, J. and Kunz, E., Der kanonische Modul eines Cohen-Macaulay-Rings, Lecture Notes in Math., 238, Springer (1971).

[ 9 ] Herzog, J. and Ulrich, B., Self-linked curve singularities, Nagoya Math. J., 120 (1990), 129-153.

[10] Huckaba, S., Analytic spread modulo an element and symbolic Rees algebras, J. Algebra, 128 (1990), 306-320.

[11] Huneke, C., Hilbert functions and symbolic powers, Michigan Math. J., 34 (1987), 293-318.

[12] — , The primary components of and integral closures of ideals in 3-dimensional regular local rings, Math. Ann., 275 (1986), 617-635.

[13] —-, On the finite generation of symbolic blow-ups, Math. Z., 179 (1982), 465-472.

[14] McAdam, S., Asymptotic prime divisors, Lecture Notes in Math., 1023, Springer (1983).

[15] Morimoto, M. and Goto, S., Non-Cohen-Macaulay symbolic blow-ups for space monomial curves, to appear in Proc. Amer. Math. Soc.

[16] Peskine, C. and Szpiro, L., Liaison des variétés algébliques. I., Invent Math., 26 (1975), 271-302.

[17] Sally, J., Numbers of generators of ideals in local rings, Lecture Notes in Pure and Appl. Math., 35, Marcel Dekker (1978).

[18] Serre, J. P., Algèbre Locale: Multiplicités (third edition), Lecture Notes in Math., 11, Springer Verlag (1975).

[19] Schenzel, P., Examples of Noetherian symbolic blow-up rings, Rev. Roumaine Math. Pures Appl., 33 (1988), 4, 375-383.

[20] Simis, A. and Trung, N. V., The divisor class group of ordinary and symbolic blow-ups, Math. Z., 198 (1988), 479-491.

[21] Valla, G., On the symmetric and Rees algebras of an ideal, manuscripta math., 30 (1980), 239-255.

S. Goto

Department of Mathematics

School of Science and Technology

Meiji University

Higashimita, Tama-ku

Kawasaki-shi 214

Japan 
K. Nishida

Department of Mathematics

Faculty of Science

Chiba University

Yayoi-cho, Chiba-shi 260

Japan

Y. Shimoda

College of Liberal Arts and Sciences

Kitasato University

Kitasato, Sagamihara-shi 228

Japan 